\title{
COMPATIBLE SYSTEMS OF GALOIS REPRESENTATIONS ASSOCIATED TO THE EXCEPTIONAL GROUP $E_{6}$
}

\author{
GEORGE BOXER $^{1}$, FRANK CALEGARI ${ }^{1}$, MATTHEW EMERTON $^{1}$, \\ BRANDON LEVIN $^{2}$, KEERTHI MADAPUSI PERA ${ }^{3}$ and STEFAN PATRIKIS ${ }^{4}$ \\ ${ }^{1}$ Department of Mathematics, University of Chicago, 5734 S University Ave Chicago, \\ IL 60637, USA; \\ email: gboxer@math.uchicago.edu, fcale@math.uchicago.edu, emerton@math.uchicago.edu \\ ${ }^{2}$ Department of Mathematics, University of Arizona, 617 N. Santa Rita Avenue, Tucson, \\ Arizona 85721, USA; \\ email: bwlevin@math.arizona.edu \\ ${ }^{3}$ Department of Mathematics, Boston College, Chestnut Hill, MA 02467, USA; \\ email: keerthi.madapusipera@bc.edu \\ ${ }^{4}$ Department of Mathematics, The University of Utah, 155 S 1400 E, Salt Lake City, \\ UT 84112, USA; \\ email: patrikis@math.utah.edu
}

Received 22 May 2018; accepted 11 October 2018

\begin{abstract}
We construct, over any CM field, compatible systems of $l$-adic Galois representations that appear in the cohomology of algebraic varieties and have (for all $l$ ) algebraic monodromy groups equal to the exceptional group of type $E_{6}$.

2010 Mathematics Subject Classification: 11F70, 11F80 (primary); 20G41 (secondary)
\end{abstract}

\section{Introduction}

In [Ser94], Serre raised the question of whether $G_{2}$ or $E_{8}$ was the motivic Galois group of a motive $M$ over a number field, and one can evidently ask the same question for the other exceptional simple Lie groups. A slightly weaker version of this question asks for a motive $M$ such that the associated $p$-adic Galois representations have algebraic monodromy group equal to the exceptional

(c) The Author(s) 2019. This is an Open Access article, distributed under the terms of the Creative Commons Attribution licence (http://creativecommons.org/licenses/by/4.0/), which permits unrestricted re-use, distribution, and reproduction in any medium, provided the original work is properly cited. 
group in question. In this form, Serre's question was answered in the affirmative by Yun in [Yun14], who also dealt with the case of the exceptional group $E_{7}$. (A stronger version of the question for the group $G_{2}$ had previously been answered by Dettweiler and Reiter [DR10].) This left open the cases of $E_{6}$ or $F_{4}$. In [Pat16], the last author of this paper succeeded in constructing geometric Galois representations for the remaining exceptional groups using arguments inspired by Ramakrishna's lifting theorems [Ram02], at least for a set of primes $p$ of density one (improved to all but finitely many in [Pat17, Theorem 1.2]). While this answered a weak form of (the $E_{6}$ analogue of) Serre's question, the Galois representations constructed in [Pat16, Pat17] did not obviously come from motives $M$ or from compatible systems of Galois representations (although that would certainly be a consequence of the Fontaine-Mazur conjectures [FM95]). The main goal of this paper is to remedy this lacuna for the group $E_{6}$.

THEOREM 1.1. Let $F / F^{+}$be a totally imaginary CM field with maximal totally real subfield $F^{+}$. Let $G$ denote the simply connected form of $E_{6}$, and fix a minuscule representation $G \rightarrow \mathrm{GL}_{27}$. Then there exists a strongly compatible system of Galois representations with coefficients in a number field $M$ such that the representations

$$
r_{\lambda}: G_{F} \rightarrow G\left(\bar{M}_{\lambda}\right) \hookrightarrow \mathrm{GL}_{27}\left(\bar{M}_{\lambda}\right)
$$

have images with Zariski closure $G\left(\bar{M}_{\lambda}\right)$ for all primes $\lambda$. Moreover, this compatible system is potentially automorphic and motivic in the sense that there is a CM extension $H / F$ such that:

- There a cuspidal automorphic representation $\pi$ for $\mathrm{GL}_{27} / H$ such that $\left.r_{\lambda}\right|_{G_{H}}$ is the compatible system of Galois representations associated to $\pi$.

- The compatible system $r_{\lambda}$ satisfies the conclusion of the Fontaine-Mazur conjecture: there is a smooth projective variety $X / F$ and integers $i$ and $j$ such that $r_{\lambda}$ is a $G_{F}$-subrepresentation of $H^{i}\left(X_{\bar{F}}, \overline{\mathbf{Q}}_{l}(j)\right)$.

The main idea of the paper is to follow the strategy of [Pat16], but to replace the lifting theorems inspired by Ramakrishna with those inspired by the work of Khare and Wintenberger [KW09a, KW09b], exploiting the modularity lifting theorems of [BLGGT14]. In order to do this, one must link $G$-representations with $\mathrm{GL}_{n}$ representations by choosing some (faithful) representation $r: G \rightarrow \mathrm{GL}_{n}$. The methods of [BLGGT14] require that the corresponding Galois representations have distinct Hodge-Tate weights. This imposes a strong restriction on the representation $r$, namely, that its formal character should be multiplicity-free. In particular, the method of this paper only 
applies to the exceptional groups $G_{2}, E_{6}$, and $E_{7}$, in their quasiminuscule $\left(G_{2}\right)$ or minuscule ( $E_{6}$ and $E_{7}$ ) representations (we concentrate on $E_{6}$ because other methods are available in the other cases). As in [Pat16, Pat17], we require a seed representation $\bar{\rho}: G_{F} \rightarrow G\left(\overline{\mathbf{F}}_{p}\right)$ from which to construct geometric lifts. In [Pat16], suitable representations $\bar{\rho}$ came from composing representations associated to modular forms with the principal $\mathrm{SL}_{2}$. These representations are not suitable for our purposes, because their composition with the minuscule representation is reducible. Instead, we construct a representation related to the action of the Weyl group of $E_{6}$ on the weight space of our representation. The fact that the representation we consider is irreducible in $\mathrm{GL}_{n}$ relies on the assumption that $r$ is minuscule. The reason we succeed in controlling the monodromy groups at all primes is a consequence of elementary combinatorial properties of the formal character of $E_{6}$ (using ideas of Larsen and Pink [LP92]) together with our ability to exploit independence of $p$ results in compatible systems of Galois representations associated to automorphic forms [TY07, Shi11, Car12].

We end the introduction with some remarks on what the methods of this paper cannot do.

REMARK 1.2 (Galois representations for $\mathbf{Q}$ versus imaginary quadratic fields). Our construction gives compatible systems of $E_{6}$-representations over any imaginary quadratic field $F$; these extend to representations of $G_{\mathbf{Q}}$ whose image is Zariski-dense in the L-group $G \rtimes \operatorname{Out}(G)$ of an outer form of $E_{6}$. We leave open the question as to whether actual $E_{6}$-systems exist over $\mathbf{Q}$, noting that the methods of this paper will not succeed in constructing them. Indeed, our methods require that the corresponding Galois representations have regular weight, and there do not exist any such compatible systems of Galois representations over $\mathbf{Q}$ (see Remark 5.1).

REMARK 1.3 (Motives versus motives with coefficients). We ultimately construct compatible systems of 27-dimensional Galois representations for a coefficient field $L$ over which we have little control. One can ask the more refined question of whether there exists a motive $M$ with coefficients over $\mathbf{Q}$ of type $E_{6}$ (Yun's result [Yun14] answer this question in the affirmative for $E_{8}$ ). One reason that this refinement is interesting is that it would have applications to the inverse Galois problem (see Corollary 6.1).

It seems to us that the Galois theoretic methods of either this paper or of [Pat16, Pat17] are unsuited to answering such a question. It is illustrative to consider the simpler case of $\mathrm{GL}_{2}$. By constructing a geometric Galois representation $\rho: G_{\mathbf{Q}} \rightarrow \mathrm{GL}_{2}\left(\mathbf{Q}_{p}\right)$, one can hope to prove it is automorphic and hence associated to a modular form $f$, and thus to construct a corresponding motive $M_{f}$ [Sch90]. 
But it seems very hard to impose conditions on $\rho$ to ensure that the form $f$ has coefficients in $\mathbf{Q}$. For example, in weight 2 (on the modular form side) one would want to put conditions on $\rho$ to ensure that it actually come from an elliptic curve rather than an abelian variety of $\mathrm{GL}_{2}$-type. In practice, we actually work in highly regular weight, and there is a certain amount of numerical evidence [Rob17] pointing to the fact that there may not exist any motives $M$ at all with coefficients in $\mathbf{Q}$ and monodromy group $\mathrm{GL}_{2}$ with Hodge-Tate weights $[0, k-1]$ when $k>50$.

\section{The $\bmod p$ representation}

In this section, we construct the mod $p$ representations that we lift in the next section using potential automorphy theorems.

DEFINITION 2.1. Let $G$ be the split simply connected reductive group scheme over $\mathbf{Z}$ of type $E_{6}$. Fix a pinned based root datum of $G$, and let ${ }^{L} G=G \rtimes \operatorname{Out}(G)$ with the nontrivial element $\tau$ of $\operatorname{Out}(G)=\mathbf{Z} / 2 \mathbf{Z}$ acting through the corresponding pinned automorphism. The induced action of $\tau$ on $W_{G}$ is conjugation by the longest element $w_{0}$, since $\tau$ acts on $T$, the maximal torus of the pinning, by the opposition involution $-w_{0}$. Let $W=W_{G} \rtimes \operatorname{Out}(G)$, and continue to write $\tau$ for the nontrivial element of $\operatorname{Out}(G) \subset W$. We write $B$ for the Borel subgroup of $G$ associated to the based root datum.

REMARK 2.2. There is an isomorphism $W \simeq W_{G} \times \mathbf{Z} / 2 \mathbf{Z}$ given by the identity on $W_{G}$ and sending $\tau$ to $\left(w_{0}, 1\right)$.

We fix, once and for all, a choice of minuscule representation $r_{\min }: G \rightarrow \mathrm{GL}_{27}$, writing $\Lambda_{\min }$ for the weights of $T$ in $r_{\min }$. Now we explain how to extend $r_{\min }$ to ${ }^{L} G$. Let $\mathcal{G}_{27}=\left(\mathrm{GL}_{27} \times \mathrm{GL}_{1}\right) \rtimes \mathbf{Z} / 2 \mathbf{Z}$, where the nontrivial element $J \in \mathbf{Z} / 2 \mathbf{Z}$ acts via $J(g, \mu) J^{-1}=\left(\mu \cdot{ }^{t} g^{-1}, \mu\right)$. The representation $g \mapsto r_{\min }\left(\tau g \tau^{-1}\right)$ is isomorphic to the dual minuscule representation of $G$, so there exists $A \in \mathrm{GL}_{27}$ such that $r_{\min }\left(\tau g \tau^{-1}\right)=A \cdot{ }^{t} r_{\min }(g)^{-1} A^{-1}$. Iterating, we see that $A \cdot{ }^{t} A^{-1}$ commutes with $r_{\min }$, so must be a scalar: $A={ }^{t} A \cdot \varepsilon$. Clearly $\varepsilon \in\{ \pm 1\}$, and since 27 is odd, we must in fact have $\varepsilon=1$ by considering determinants.

We can now extend $r_{\text {min }}$ to

$$
r_{\min }:{ }^{L} G \rightarrow \mathcal{G}_{27}
$$

by $r_{\min }(\tau)=(A, \varepsilon, J)=(A, 1, J)$. The fact that $\varepsilon=1$ has the following consequence, which we need later: 
LEMMA 2.3. Let $v: \mathcal{G}_{27} \rightarrow \mathbf{G}_{m}$ be the character given by $v(g, a, 0)=a$, $v(j)=-1$. Let $x \in{ }^{L} G$ be any element with nontrivial projection to $\operatorname{Out}(G)$ (the case of interest will be $x$ such that conjugation by $x$ induces a Chevalley involution of $G$ ). Then $v \circ r_{\min }(x)=-1$.

LEMMA 2.4. Let $E^{+} / F^{+}$be a Galois extension of totally real fields whose Galois group is identified with a subgroup $P$ of $W_{G}$, and let $F / F^{+}$be a quadratic totally imaginary extension. Let $E=F . E^{+}$. Then the composite

$\operatorname{Gal}\left(E / F^{+}\right) \stackrel{\sim}{\rightarrow} \operatorname{Gal}\left(E^{+} / F^{+}\right) \times \operatorname{Gal}\left(F / F^{+}\right) \stackrel{\sim}{\rightarrow} P \times \mathbf{Z} / 2 \mathbf{Z} \subset W_{G} \times \mathbf{Z} / 2 \mathbf{Z} \stackrel{\sim}{\rightarrow} W$

sends complex conjugation to $\left(w_{0}, \tau\right) \in W=W_{G} \rtimes \operatorname{Out}(G)$. In this setting we write ${ }^{L} P$ for the image of $P \times \mathbf{Z} / 2 \mathbf{Z}$ in $W$.

Proof. This follows from the definition of the isomorphism $W_{G} \times \mathbf{Z} / 2 \mathbf{Z} \stackrel{\sim}{\rightarrow} W$ (Remark 2.2).

We now construct the mod $p$ Galois representation. Let $P$ be a Sylow 3subgroup of $W_{G}$; we note that $\left|W_{G}\right|=2^{7} \cdot 3^{4} \cdot 5$. Let $F / F^{+}$denote a fixed totally imaginary quadratic extension of our fixed totally real field $F^{+}$. Our starting point will be to construct $P$ as a Galois group over $F^{+}$, with some local restrictions whose significance will become apparent when we apply potential automorphy theorems.

Lemma 2.5. There exists a totally real Galois extension $E^{+} / F^{+}$together with an equality $\operatorname{Gal}\left(E^{+} / F^{+}\right)=P$ (we write ' $=$ ' to denote a fixed isomorphism) and all the primes of $F^{+}$which ramify in $E^{+}$are split in $F / F^{+}$.

Proof. The Scholz-Reichardt theorem [Ser08, Theorem 2.1.1] guarantees the existence of a number field $L$ with $\operatorname{Gal}(L / \mathbf{Q})=P$, and, because $|P|$ is odd, such an extension will automatically be totally real. It suffices to show that we can construct such an extension ramified only at primes which are totally split in $F$ (equivalently, in the Galois closure of $F$ ). This forces the intersection of $L$ with the Galois closure of $F$ to be unramified everywhere over $\mathbf{Q}$ and hence trivial, and thus $E^{+}=L . F^{+}$will have Galois group $P$ over $F$ and produce the desired extension. The result follows immediately by induction and from the following lemma, which is extremely close to [Ser08, Theorem 2.1.3]:

Sublemma 2.6. Let $\widetilde{A} \rightarrow A$ be a central extension of a finite group $A$ by $\mathbf{Z} / p \mathbf{Z}$, and assume that the exponent of $\widetilde{A}$ divides $p^{n}$. Let $L / \mathbf{Q}$ be Galois with Galois 
group $A$, and assume that every prime $l$ which ramifies in $L$ has the following properties:

(1) $l \equiv 1 \bmod p^{n}$.

(2) The inertia group(s) of $l$ in A coincides with the decomposition group $(s)$ at $l$.

(3) $l$ splits completely in $F$.

Then there exists an extension $\widetilde{L} / L$ which is Galois over $\mathbf{Q}$ with Galois group $\widetilde{A}$ and such that the primes $l$ which ramify in $\widetilde{L}$ satisfy the same conditions as above.

The only difference between this statement and [Ser08, Theorem 2.1.3] is the extra requirement that the primes $l$ splits completely in $F$. There are two inductive steps in the proof of Theorem 2.1.3 of [Ser08], and we indicate the required argument to show that the new auxiliary prime $q$ may be chosen to split completely in $F$.

Suppose first that $\widetilde{A}=A \times \mathbf{Z} / p \mathbf{Z}$ is a split extension. Pick any prime $q \equiv 1$ mod $p^{n}$ which is totally split in the Galois closure of $F$ and also totally split in the field $L\left(\zeta_{p^{n}},\left\{l^{1 / p}\right\}_{l \in \operatorname{Ram}(L / \mathbf{Q})}\right)$ given by adjoining the $p$ th roots of primes $l$ which ramify in $L$. Then take $\widetilde{L}$ to be the composite of $L$ and the subextension of $\operatorname{Gal}\left(\mathbf{Q}\left(\zeta_{q}\right) / \mathbf{Q}\right)$ with Galois group $\mathbf{Z} / p \mathbf{Z}$.

Now suppose that $\widetilde{A}$ is a nonsplit extension. The argument in [Ser08] proceeds by first finding an extension $\widetilde{L}$ and then modifying $\widetilde{L}$ so that it is ramified at the same places as $L$. Hence the ramified primes $l$ automatically satisfy the required splitting condition in $F$. The final step is to modify the field further so that it has property (2). This is achieved by choosing an auxiliary prime $q \equiv 1 \bmod p$ along with a character $\chi:(\mathbf{Z} / q \mathbf{Z})^{\times} \rightarrow \mathbf{Z} / p \mathbf{Z}$ satisfying the following properties:

(1) The prime $q \equiv 1 \bmod p^{n}$.

(2) For every prime $l$ which ramifies in $L$, there is an equality $\chi(l)=c_{l}$ where $c_{l}$ is determined from the extension $L$.

(3) The prime $q$ splits completely in $L$.

(4) The prime $q$ splits completely in $F$.

Only the last condition is new. The first three conditions are Čebotarev conditions in the field $L\left(\zeta_{p^{n}},\left\{l^{1 / p}\right\}_{l \in \operatorname{Ram}(L / \mathbf{Q})}\right)$, whereas the fourth condition is a Čebotarev condition in the Galois closure of $F$. By construction, the primes $l$ are totally split in the Galois closure of $F$. Hence the intersection of the Galois closure of $F$ with $L\left(\zeta_{p^{n}},\left\{l^{1 / p}\right\}_{l}\right)$ must be contained inside $\mathbf{Q}\left(\zeta_{p^{n}}\right)$. Since the first condition implies 
that $q$ splits completely in $\mathbf{Q}\left(\zeta_{p^{n}}\right)$, there is no obstruction to finding such primes $q$ satisfying all four conditions using the Čebotarev density theorem provided there is no obstruction without the last hypothesis. But this is exactly what follows from the proof of Theorem 2.1.3 of [Ser08].

As in Lemma 2.4, let ${ }^{L} P$ be the image of $P \times \mathbf{Z} / 2 \mathbf{Z}$ in $W$; explicitly, it is the product $P \times\left\langle\left(w_{0}, \tau\right)\right\rangle$ inside $W$. Our reason for working with the group $P$ is the combination of the following two properties:

LEMMA 2.7. (1) The restriction of the extension

$$
1 \rightarrow T(\mathbf{Z}) \rightarrow N_{G}(T)(\mathbf{Z}) \rtimes \operatorname{Out}(G) \stackrel{\pi}{\rightarrow} W_{G} \rtimes \operatorname{Out}(G) \rightarrow 1
$$

to ${ }^{L} P$ splits.

(2) $P$ acts transitively on the set $\Lambda_{\min }$ of weights of the minuscule representation $r_{\min }$.

Proof. Since $P$ is a 3-group while $T(\mathbf{Z})$ is an $\mathbf{F}_{2}$-module, the Hochschild-Serre spectral sequence

$$
H^{i}\left(P, H^{j}\left(\left\langle\left(w_{0}, \tau\right)\right\rangle, T(\mathbf{Z})\right)\right) \Longrightarrow H^{i+j}\left({ }^{L} P, T(\mathbf{Z})\right)
$$

degenerates at the $E_{2}$-page, and restriction induces an isomorphism

$$
H^{2}\left({ }^{L} P, T(\mathbf{Z})\right) \stackrel{\sim}{\rightarrow} H^{2}\left(\left\langle\left(w_{0}, \tau\right)\right\rangle, T(\mathbf{Z})\right)^{P} .
$$

The image of our extension under this restriction isomorphism is trivial, because the element $\left(w_{0}, \tau\right)$ lifts to an order two element of $N(T)(\mathbf{Z}) \rtimes \operatorname{Out}(G)$ (see for instance [AH16, Lemma 3.1], noting that $Z_{G}$ has order 3).

For the second part of the lemma, note that $W_{G}$ acts transitively on $\Lambda_{\min }$, so the 3 -part of the stabilizer of any element $\lambda \in \Lambda_{\min }$ has order 3 (recall $\left|W_{G}\right|=2^{7} \cdot 3^{4} \cdot 5$ and $\left.\left|\Lambda_{\min }\right|=3^{3}\right)$. It follows that the orbit of $P$ on $\lambda$ has order at least $|P| / 3=3^{3}$, and thus (equality holds and) $P$ acts transitively. (More generally, a finite group $G$ acts transitively on a set $X$ of $p$-power order if and only if a $p$-Sylow subgroup $P$ acts transitively on $X$.)

The work of Shafarevich on the inverse Galois problem for solvable groups implies that every split embedding problem with nilpotent kernel has a proper solution; we need some precise local control so will not be able to invoke this theorem, and unfortunately the following construction, guided by the demands of the local deformation theory as in [Pat16] and of automorphy lifting as in [BLGGT14], is somewhat technical. 
Applying Lemma 2.7, let us fix a splitting of the extension

$$
1 \rightarrow T(\mathbf{Z}) \rightarrow \pi^{-1}\left({ }^{L} P\right) \stackrel{\pi}{\rightarrow}{ }^{L} P \rightarrow 1
$$

and write $s: \operatorname{Gal}\left(E / F^{+}\right) \rightarrow \pi^{-1}\left({ }^{L} P\right)$ for the resulting lift. This representation is not yet suitable for potential automorphy theorems, so we modify it in the following Proposition.

We first establish some notation. For a fixed prime $p$, we write $\epsilon$ for the $p$ adic cyclotomic character and $\bar{\epsilon}$ for its $\bmod p$ reduction. For a prime $l$ exactly dividing $p-1$, let $\operatorname{pr}(l)$ be the projection from $\mathbf{F}_{p}^{\times}$onto the $l$-torsion subgroup $\mathbf{F}_{p}^{\times}[l]$ restricting to the identity on $\mathbf{F}_{p}^{\times}[l]$. Finally, let $\bar{\epsilon}[l]=\operatorname{pr}(l) \circ \bar{\epsilon}: G_{F^{+}} \rightarrow$ $\mathbf{F}_{p}^{\times}[l]$. For any homomorphism $\bar{\rho}: G_{F^{+}} \rightarrow G\left(\mathbf{F}_{p}\right)$ and any place $v$ of $F^{+}$, let $\bar{\rho}_{v}:=\left.\bar{\rho}\right|_{G_{F_{v}^{+}}}$. Finally, for any homomorphism of groups $\rho: \Gamma \rightarrow \Gamma^{\prime}$, and any $\rho(\Gamma)$-module $M$, let $\rho(M)$ denote $M$ regarded as a $\Gamma$-module.

Proposition 2.8. Consider pairs of primes $(l, p)$ such that:

- All primes above l split in $F / F^{+}$.

- $p$ splits in $E / \mathbf{Q}$ and $p-1$ is divisible by $l$ but not by $l^{2}$.

Then there exist infinitely many primes $l$ such that there exist infinitely many pairs $(l, p)$ such that there exists a homomorphism

$$
\bar{\rho}: G_{F^{+}} \rightarrow T\left(\mathbf{F}_{p}\right)[l] \cdot \pi^{-1}\left({ }^{L} P\right) \subset N_{G}(T)\left(\mathbf{F}_{p}\right) \rtimes \operatorname{Out}(G)
$$

lifting our fixed identification $\operatorname{Gal}\left(E / F^{+}\right)={ }^{L} P$ and satisfying the following:

(1) The restriction $\left.\bar{\rho}\right|_{G_{F}}$ is ramified only at places split in $F / F^{+}$.

(2) For any choice of complex conjugation $c \in G_{F^{+}}, \bar{\rho}(c)$ is a Chevalley involution of $G$, that is, $\operatorname{dim}\left(\mathfrak{g}^{\operatorname{Ad}(\bar{\rho}(c))=1}\right)=\operatorname{dim}(G / B)=36 .{ }^{1}$

(3) For all places $v \mid p$ of $F^{+}$, fix any choice of integers $n_{v, \alpha}$ indexed by simple roots $\alpha \in \Delta=\Delta(G, B, T)$. Then $\left.\bar{\rho}\right|_{G_{F_{v}^{+}}}$is equal to

$$
\prod_{\alpha \in \Delta} \alpha^{\vee} \circ \bar{\epsilon}[l]^{n_{v, \alpha}} .
$$

(4) Let $\rho_{G}^{\vee}$ denote the half-sum of the positive coroots of $(G, B, T)$; it lies in $X_{\bullet}(T)$ since $\# Z_{G}=3$. There is a set $S_{\text {reg }}$ of two primes $q$ split in $E / \mathbf{Q}$ and of order l modulo $p$ such that for some place $v \mid q$ of $F^{+},\left.\bar{\rho}\right|_{G_{F_{v}}}$ is unramified with Frobenius mapping to $\rho_{G}^{\vee}(q)$ (which lands inside the group $T\left(\mathbf{F}_{p}\right)[l]$ because $\left.q^{l} \equiv 1(\bmod p)\right)$.

${ }^{1}$ S.P. apologizes for the nonstandard term 'split Cartan involution' used in [Pat16, Pat17]. 
Moreover, in addition to satisfying the above conditions, we can choose $p>56=$ $2 \cdot(27+1), l>h_{G}=12$ (the Coxeter number of $\left.G\right),\left\{n_{v, \alpha}\right\}$, and $\bar{\rho}$ such that

(1) For all $v \mid p$, the composite $\left.r_{\min } \circ \bar{\rho}\right|_{G_{F_{v}}}$ is a direct sum of distinct powers of $\bar{\epsilon}[l]$.

(2) For all $v \mid p$ and for any Borel subgroup $B$ (with Lie algebra $\mathfrak{b}$ ) containing $T$, the cohomology groups $H^{0}\left(G_{F_{v}^{+}}, \bar{\rho}_{v}(\mathfrak{g} / \mathfrak{b})\right)$ and $H^{0}\left(G_{F_{v}^{+}}, \bar{\rho}_{v}(\mathfrak{g} / \mathfrak{b})(1)\right)$ both vanish. Moreover, for any Borel subgroup $B_{27}$ containing the maximal torus of $\mathrm{SL}_{27}$ that stabilizes the weight spaces of $r_{\min }, H^{0}\left(G_{F_{v}^{+}},\left(r_{\min } \circ\right.\right.$ $\left.\left.\bar{\rho}_{v}\right)\left(\mathfrak{s l}_{27} / \mathfrak{b}_{27}\right)\right)=0$ and $H^{0}\left(G_{F_{v}^{+}},\left(r_{\min } \circ \bar{\rho}_{v}\right)\left(\mathfrak{s l}_{27} / \mathfrak{b}_{27}\right)(1)\right)=0$.

(3) The composite $\left.r_{\min } \circ \bar{\rho}\right|_{G_{F(\zeta p)}}$ is absolutely irreducible.

Proof. For now let $l$ be any odd prime such that the places of $F^{+}$above $l$ are all split in $F / F^{+}$, and $E$ and $\mathbf{Q}\left(\mu_{l^{2}}\right)$ are linearly disjoint over $\mathbf{Q}$ (for example, take $l$ split in $E / \mathbf{Q}$ ); later in the argument we require $l$ to be larger than some absolute bound depending only on the group $G$. Let $p$ be any prime split in $E / \mathbf{Q}$ such that $l$, but not $l^{2}$, divides $p-1$ (such $p$ exist by Čebotarev). Fix a set $S_{\text {reg }}$ of two rational primes $q$ that are split in $E / \mathbf{Q}$ and have order $l$ modulo $p$ : such $q$ exist since $E$ and $\mathbf{Q}\left(\mu_{p}\right)$ are linearly disjoint over $\mathbf{Q}$ ( $p$ splits in $E / \mathbf{Q}$ ). We modify the original lift $s: \operatorname{Gal}\left(E / F^{+}\right) \rightarrow \pi^{-1}\left({ }^{L} P\right)$ by an element of $H^{1}\left(G_{F^{+}}\right.$, $T\left(\mathbf{F}_{p}[l]\right)$ ) so as to satisfy the local conditions (here $G_{F^{+}}$continues to act via the quotient $\operatorname{Gal}\left(E / F^{+}\right)$; note that $T\left(\mathbf{F}_{p}\right)[l]$ is $\tau$-stable). Let $T\left(\mathbf{F}_{p}\right)[l]=\bigoplus W_{i}$ be the decomposition into irreducible $\mathbf{F}_{l}[P]$-modules. Recall that $\left(w_{0}, c\right) \in{ }^{L} P$ acts on $T$ by -1 , so this is also a decomposition as ${ }^{L} P$-module, and the splitting field $F^{+}\left(W_{i}\right)$ of the $\operatorname{Gal}\left(E / F^{+}\right)$-module $W_{i}$ contains $F$ for each $i$ (since $l \neq 2$ ). Let $\Sigma$ be the set of places of $F^{+}$that are either split or ramified in $F / F^{+}$(implicitly including the infinite places in the former condition); in particular, the action of $G_{F^{+}}$on $T\left(\mathbf{F}_{p}\right)[l]$ factors through $G_{F^{+}, \Sigma}$. For any finite subset $T$ of $\Sigma$, [NSW08, Theorem 9.2.3(v)] implies that the restriction map

$$
H^{1}\left(G_{F^{+}, \Sigma}, W_{i}\right) \rightarrow \bigoplus_{T} H^{1}\left(G_{F_{v}^{+}}, W_{i}\right)
$$

is surjective for all $i$. Assembling the different $i$ (with a common set $T$ ), the restriction map

$$
H^{1}\left(G_{F^{+}, \Sigma}, T\left(\mathbf{F}_{p}\right)[l]\right) \rightarrow \bigoplus_{T} H^{1}\left(G_{F_{v}^{+}}, T\left(\mathbf{F}_{p}\right)[l]\right)
$$

is also surjective. We apply this observation to the following set $T$ and the following local cohomology classes: let $T$ be the union of the following sets of places of $F^{+}$: 
- places which are ramified in $F / F^{+}$;

- places dividing $p$;

- places above the rational primes $q \in S_{\text {reg; }}$;

- an auxiliary place $w$ lying above a rational prime $r$ that splits completely in $E\left(\zeta_{p}\right) / \mathbf{Q}$.

Consider the following local classes in $H^{1}\left(G_{F_{v}^{+}}, T\left(\mathbf{F}_{p}\right)[l]\right)$ for $v \in T$ :

- trivial at places which ramify in $F / F^{+}$;

- the prescribed homomorphism $\prod_{\alpha \in \Delta} \alpha^{\vee} \circ \bar{\epsilon}[l]^{n_{v, \alpha}}$ for $v \mid p$,

- the unramified homomorphism $\operatorname{Fr}_{v} \mapsto \rho_{G}^{\vee}(q)$ for $v \mid q$ and $q \in S_{\text {reg }}$, where $\rho_{G}^{\vee}$ denotes the half-sum of the positive coroots of $G$, which is in fact a cocharacter of $G$ (we only need this construction for one of the places above $q$ ).

- the unramified homomorphism $\operatorname{Fr}_{w} \mapsto t$, where $t$ is any element of $T\left(\mathbf{F}_{p}\right)[l]$ such that the values $\lambda(t)$ for $\lambda \in \Lambda_{\min }$ are all distinct (for $l$ sufficiently large, such $t$ exist).

Let $\phi \in H^{1}\left(G_{F^{+}, \Sigma}, T\left(\mathbf{F}_{p}\right)[l]\right)$ be a class with these local restrictions, and set $\bar{\rho}=\phi \cdot s$. We claim the conclusions of the proposition hold for this $\bar{\rho}$. The conditions at finite places are all evident from the construction, and the condition on complex conjugation is satisfied because any order two element $\left(\widetilde{w}_{0}, \tau\right) \in$ $N_{G}(T)\left(\mathbf{F}_{p}\right)$ lifting $\left(w_{0}, \tau\right) \in W_{G} \rtimes \operatorname{Out}(G)$ gives a Chevalley involution of $\mathfrak{g}$ : it acts by -1 on $\operatorname{Lie}(T)$, and it sends a root space $\mathfrak{g}_{\alpha}$ to $\mathfrak{g}_{-\alpha}$, so we get precisely $\operatorname{dim}(G / B)=\operatorname{dim}\left(\mathfrak{g}^{\operatorname{Ad}\left(\left(\widetilde{w}_{0}, \tau\right)\right)=1}\right)$.

For the second list of assertions, note that for $v\left|p, r_{\min } \circ \bar{\rho}\right|_{G_{F_{v}^{+}}}$is equal to

$$
\bigoplus_{\lambda \in \Lambda_{\min }} \bar{\epsilon}[l]^{\sum_{\alpha \in \Delta} n_{v, \alpha}\left\langle\lambda, \alpha^{\vee}\right\rangle},
$$

where $\Lambda_{\min }$ is the set of weights of $r_{\min }$. For varying $\lambda$, we want these exponents to be distinct modulo $l$. We simply choose the $\left(n_{v, \alpha}\right)_{\alpha}$ so that the exponents are distinct in $\mathbf{Z}$, and then any $l$ sufficiently large will do. To evaluate the cohomology groups appearing in the conclusion of the proposition, note that as a $G_{F_{v}^{+}}$-module, $\bar{\rho}_{v}(\mathfrak{g} / \mathfrak{b})$ is a direct sum of characters of the form

$$
\bar{\epsilon}[l]^{\sum_{\alpha \in \Delta} n_{v, \alpha}\left\langle\beta, \alpha^{\vee}\right\rangle},
$$

where $\beta$ is a negative root of $G$. Clearly the choice of $l$ can be modified if necessary to ensure that these exponents (which do not depend on $p$ ) are all 
(distinct, nonzero) integers in $(-l, l)$, and so regardless of how $p$ is chosen the group $H^{0}\left(G_{F_{v}^{+}}, \bar{\rho}_{v}(\mathfrak{g} / \mathfrak{b})\right)$ will vanish. (Since $\bar{\rho}_{v}$ factors through $T$, this argument in fact shows that $\bar{\rho}_{v}(\mathfrak{g} / \mathfrak{t})$ has no invariants; in particular, it applies to all Borel subgroups $B$ containing $T$.) The vanishing of $H^{0}\left(G_{F_{v}^{+}}, \bar{\rho}_{v}(\mathfrak{g} / \mathfrak{b})(1)\right)$ is even more straightforward: the order of $\bar{\epsilon}$ is divisible by some prime other than $l$, whereas all powers of $\bar{\epsilon}[l]$ have order 1 or $l$. We can similarly deduce the vanishing of $H^{0}\left(G_{F_{v}^{+}},\left(r_{\min } \circ \bar{\rho}_{v}\right)\left(\mathfrak{s l}_{27} / \mathfrak{b}_{27}\right)\right)$ and $H^{0}\left(G_{F_{v}^{+}},\left(r_{\min } \circ \bar{\rho}_{v}\right)\left(\mathfrak{s l}_{27} / \mathfrak{b}_{27}\right)(1)\right)$, where $\mathfrak{b}_{27}$ is the Lie algebra of any Borel subgroup $B_{27} \subset \mathrm{SL}_{27}$ containing the maximal torus of $\mathrm{SL}_{27}$ characterized by the property that it stabilizes each of the weight spaces of $r_{\text {min }}$. Then $\left(r_{\min } \circ \bar{\rho}_{v}\right)\left(\mathfrak{s l}_{27} / \mathfrak{b}_{27}\right)$ is a direct sum of characters

$$
\bar{\epsilon}[l]^{\sum_{\alpha \in \Delta} n_{v, \alpha}\left\langle\lambda_{1}-\lambda_{2}, \alpha^{\vee}\right\rangle},
$$

for distinct weights $\lambda_{1}, \lambda_{2} \in \Lambda_{\min }$. These exponents are by construction nonzero modulo $l$, so we win, and the same argument as for $\bar{\rho}_{v}(\mathfrak{g} / \mathfrak{b})(1)$ applies to show $H^{0}\left(G_{F_{v}^{+}},\left(r_{\min } \circ \bar{\rho}_{v}\right)\left(\mathfrak{s l}_{27} / \mathfrak{b}_{27}\right)(1)\right)$ is zero as well.

The condition at the auxiliary prime $w$ ensures absolute irreducibility of $r_{\min } \circ$ $\left.\bar{\rho}\right|_{G_{F(\zeta p)}}: P$ acts transitively on $\Lambda_{\text {min }}$ by Lemma $2.7(2)$, so any nonzero submodule of $\left.r_{\text {min }} \circ \bar{\rho}\right|_{G_{F(\zeta p)}}$ has nonzero projection to each weight space (recall that $F\left(\zeta_{p}\right)$ is linearly disjoint from $E$ over $F$ ); but for a place $w^{\prime} \mid w$ of $F\left(\zeta_{p}\right)$, the image of $G_{F\left(\zeta_{p}\right)_{w^{\prime}}}$ in $T\left(\mathbf{F}_{p}\right)$ acts via distinct characters on the different weight spaces of $r_{\min }$.

\section{Lifting Galois representations}

Let $\bar{\rho}: G_{F^{+}} \rightarrow N_{G}(T)\left(\mathbf{F}_{p}\right) \rtimes \operatorname{Out}(G) \subset{ }^{L} G\left(\mathbf{F}_{p}\right)$ be a homomorphism constructed as in Proposition 2.8. We would like to lift $\bar{\rho}$ to a homomorphism

$$
\rho: G_{F^{+}} \rightarrow{ }^{L} G\left(\overline{\mathbf{Z}}_{p}\right)
$$

that belongs to a compatible system of representations, all having Zariski-dense image and appearing in the cohomology of an algebraic variety. To achieve Zariski-dense monodromy for a lift $\rho$, we follow the approach of [Pat16]: ensuring local Steinberg-type ramification at one auxiliary prime and sufficiently general Hodge-Tate cocharacter suffices. To produce the lift, and to put it in a compatible system, we compare deformation rings for $\bar{\rho}$ and $r_{\min } \circ \bar{\rho}: G_{F^{+}} \rightarrow$ $\mathcal{G}_{27}\left(\mathbf{F}_{p}\right)$. We control a suitable deformation ring for $r_{\min } \circ \bar{\rho}$ using the method of Khare-Wintenberger and the automorphy lifting results of [BLGGT14].

Let $S$ be a finite set of primes of $F^{+}$which split in $F$ containing all those where $\bar{\rho}$ is ramified (by the construction of $\bar{\rho}$, the primes of $F^{+}$at which $\bar{\rho}$ is ramified split in $F$ ). We enlarge the set $S$ as necessary. Let $F_{S}$ be the maximal extension 
(in $\bar{F}^{+}$) of $F$ unramified outside (places above) $S$, and set $G_{S}=\operatorname{Gal}\left(F_{S} / F^{+}\right)$. We will be deforming $G_{S}$-representations. For each $v \in S$, fix an extension $\tilde{v}$ of $v$ to $F$, and fix a member of the $G_{F, S}$-conjugacy class of homomorphisms $G_{F_{\tilde{v}}} \rightarrow G_{F, S}$. Via the inclusion $G_{F, S} \subset G_{S}$, these choices specify what we mean by restricting $\bar{\rho}$ (or its lifts) to $G_{F_{v}}$. We do not review in detail the mechanics of the deformation theory in this setting, but instead refer the reader to [Pat16, Section 9.2] (for ${ }^{L} G$ ) and [CHT08, Section 2] (for $\mathcal{G}_{27}$ ). We now specify local deformation conditions to define two global deformation functors, one for $\bar{\rho}$ and one for $r_{\min } \circ \bar{\rho}$.

For $v \in S$, define the following local deformation conditions $\mathcal{P}_{v}$ on lifts of $\left.\bar{\rho}\right|_{G_{F_{\tilde{v}}}}$ :

- For $v$ above the auxiliary primes $q \in S_{\text {reg }}$ (see Proposition 2.8), we impose the Steinberg deformation condition as in [Pat16, Section 4.3], with respect to the Borel subgroup $B$ of $G$ specified by our based root datum. Note that by construction the order of $\bar{\epsilon}: G_{F_{\tilde{v}}} \rightarrow \mathbf{F}_{p}^{\times}$is greater than $h_{G}-1$ (since $l>h_{G}$ is the order of $q$ modulo $p$ ).

- For $v \mid p$, we take an ordinary deformation condition as in [Pat16, Section 4.1]. To be precise, fix the following lift $\chi_{T}$ of $\left.\bar{\rho}\right|_{I_{\tilde{v}}}$ to $T\left(\mathbf{Z}_{p}\right)$ :

$$
\chi_{T}=\prod_{\alpha \in \Delta} \alpha^{\vee} \circ\left(\epsilon^{\tilde{n}_{v, \alpha}} \cdot \chi^{-n_{v, \alpha}}\right),
$$

where the $\tilde{n}_{v, \alpha}$ are sufficiently general positive (positive ensures, as in [Pat16, Lemma 4.8], that our characteristic zero lifts are de Rham) integers congruent to $n_{v, \alpha}$ modulo $l-1$, and $\chi$ is the Teichmüller lift of $\bar{\epsilon} \cdot \bar{\epsilon}[l]^{-1}$.

- For all other primes $v \in S$, the inertial image $\bar{\rho}\left(I_{F_{\tilde{v}}}\right)$ has order prime to $p$ (indeed, $\bar{\rho}$ lands in the prime-to- $p$ group $N_{G}(T)\left(\mathbf{F}_{p}\right) \rtimes \operatorname{Out}(G)$ ), and we take the minimal deformation condition of [Pat16, Section 4.4].

Lemma 3.1. For all $v \in S$, let $\mathcal{P}_{v}$ be the local condition just defined. Let $\mathcal{P}=$ $\left\{\mathcal{P}_{v}\right\}_{v \in S}$, and let $\operatorname{Lift}_{\bar{\rho}}^{\mathcal{P}}$ be the associated global lifting functor associated to this collection of local conditions (see [Pat16, Section 9.2]).

(1) For all $v \in S$ not above $p$, the local lifting ring associated to the condition $\mathcal{P}_{v}$ just defined is formally smooth, and the associated deformation functor has tangent space $L_{v}$ of dimension $\operatorname{dim} H^{0}\left(G_{F_{\tilde{v}}}, \bar{\rho}(\mathfrak{g})\right)$. For $v \mid p$, the same holds, except the tangent space has dimension $\operatorname{dim} H^{0}\left(G_{F_{\tilde{v}}}, \bar{\rho}(\mathfrak{g})\right)+\operatorname{dim}(G / B)$.

(2) The associated deformation functor $\operatorname{Def}_{\bar{\rho}}^{\mathcal{P}}$ is representable. Let $R_{\bar{\rho}}^{\mathcal{P}}$ be the representing object. For some integer $\delta, R_{\bar{\rho}}^{\mathcal{P}}$ has a presentation as the 
quotient of a power series ring over $\mathbf{Z}_{p}$ in $\delta$ variables by an ideal generated by (at most) $\delta$ relations.

Proof. The local claims follow from [Pat16, Sections 4.1, 4.3, 4.4]. The global claims follow from [Pat16, Proposition 9.2]), using that:

- the centralizer of $\bar{\rho}$ in $\mathfrak{g}$ is trivial;

- for all complex conjugations $c, \bar{\rho}(c)$ is a Chevalley involution of $G$;

- the local lifting rings have dimensions as computed in the first part of the lemma.

(We remark that the integer $\delta$ is the common dimension of the Selmer and dual Selmer groups associated to the global deformation functor.)

Next we define an analogous deformation ring for $r_{\min } \circ \bar{\rho}: G_{S} \rightarrow \mathcal{G}_{27}\left(\mathbf{F}_{p}\right)$. Recall the character $v: \mathcal{G}_{27} \rightarrow \mathbf{G}_{m}$. The composition $v \circ\left(r_{\min } \circ \bar{\rho}\right)$ is the nontrivial character of $\operatorname{Gal}\left(F / F^{+}\right)$, and we fix $\mu: G_{F^{+}} \rightarrow \mathbf{Z}_{p}^{\times}$equal to its Teichmüller lift (we consider lifts with this fixed character). To define a global deformation problem in the sense of [BLGGT14, Section 1.5] (see [CHT08] for more details), we must, for each $v \in S$, choose an irreducible component $\mathcal{C}_{v}$ of the (generic fibre) lifting ring (in the case $v$ not above $p$ ) $R_{\left.r_{\min } \circ \bar{\rho}\right|_{G_{F}}}^{\square}[1 / p]$ or (in the case $v \mid p$ ) $\lim _{K} R_{\left.r_{\min } \circ \bar{\rho}\right|_{G_{\tilde{v}}},\{H\}, K-\mathrm{ss}}^{\square}[1 / p]$, where here we follow the notation of [BLGGT14]: $H$ is a collection (indexed by embeddings $F_{\tilde{v}} \rightarrow \mathbf{Q}_{p}$, but for us $F_{\tilde{v}}=\mathbf{Q}_{p}$ ) of multisets of Hodge numbers, $K$ varies over finite extensions of $F_{\tilde{v}}$, and the lifting ring in question is the one constructed by Kisin [Kis08], whose characteristic zero points parametrize potentially semistable deformations, semistable over $K$, with the prescribed Hodge numbers; to be precise, it is the maximal reduced $p$ torsion-free quotient of $R_{\left.r_{\text {min }} \circ \bar{\rho}\right|_{G_{F}}}^{\square}$ whose $\overline{\mathbf{Q}}_{p}$-points satisfy these properties (see [BLGGT14, Section 1.4] for an overview). In both cases $v \mid p$ and $v \nmid p$, we then associate the lifting ring $R_{\left.r_{\min } \circ \bar{\rho}\right|_{G_{F}}}^{\mathcal{C}_{\vec{v}}}$ given by the maximal reduced $p$-torsion-free quotient of $(v \nmid p) R_{\left.r_{\min } \circ \bar{\rho}\right|_{G_{\tilde{v}}}}^{\square}$ or $(v \mid p) \lim _{K} R_{\left.r_{\min } \circ \bar{\rho}\right|_{G_{F}},\{H\}, K-\mathrm{ss}}^{\square}$ that is, after inverting $p$, supported on the component $\mathcal{C}_{v}$. Namely, we take:

- For $v$ above the auxiliary primes $q \in S_{\text {reg }}$, recall that $\left.\bar{\rho}\right|_{G_{F_{\tilde{v}}}}$ is unramified with (arithmetic) Frobenius $\operatorname{Fr}_{\tilde{v}}$ mapping to $\rho_{G}^{\vee}(q)$. Let $\varphi: \mathrm{PGL}_{2} \rightarrow G$ be the principal homomorphism associated to our fixed pinning of $G$, so $\bar{\rho}\left(\operatorname{Fr}_{v}\right)$ equals $\varphi(\operatorname{diag}(q, 1))$. The composite $r_{\min } \circ \varphi$ decomposes as $\mathrm{S}^{16} \oplus \mathrm{S}^{8} \oplus \mathrm{S}^{0}$ (see [Gro00, Section 7]), where we write $S^{i}$ for the $i^{\text {th }}$ symmetric power of the standard representation of $\mathrm{SL}_{2}$. Consider the lift $\rho_{\tilde{v}}$ of $\left.r_{\min } \circ \bar{\rho}\right|_{G_{\tilde{v}}}$ given by $r_{16} \oplus r_{8} \oplus r_{0}$ 
where $r_{i}$ is the tame unipotent representation on the $S^{i}$ component given by the matrices

$$
\left(r_{i}\left(\operatorname{Fr}_{v}\right)\right)_{a, b}= \begin{cases}q^{i-2 a+2} & \text { if } a=b \\ 0 & \text { if } a \neq b,\end{cases}
$$

and on a topological generator $\tau_{v}$ of tame inertia by

$$
\left(r_{i}\left(\tau_{v}\right)\right)_{a, b}= \begin{cases}p & \text { if } b=a+1, \\ 0 & \text { if } b \neq a+1 .\end{cases}
$$

A quick calculation shows that, for all finite extensions $K / F_{\tilde{v}}$, we have an equality $H^{0}\left(G_{K}, \operatorname{ad}\left(\rho_{\tilde{v}}\right)(1)\right)=0$, so $\rho_{\tilde{v}}$ is a robustly smooth point of $R_{\left.r_{\min } \circ \bar{\rho}\right|_{G_{\tilde{v}}}}^{\square}[1 / p]$, in the sense of [BLGGT14, Section 1.3]. In particular, $\rho_{\tilde{v}}$ lies on a unique irreducible component of $R_{r_{\min } \circ \bar{\rho}_{G_{F_{\tilde{v}}}}^{\square}}[1 / p]$, and we take $\mathcal{C}_{v}$ to be this component.

- For $v \mid p$, let the set $H$ of Hodge-Tate weights be

$$
\left\{h_{\lambda}=\sum_{\alpha \in \Delta} \tilde{n}_{v, \alpha}\left\langle\lambda, \alpha^{\vee}\right\rangle\right\}_{\lambda \in \Lambda_{\min }},
$$

where $\Lambda_{\min }$ is the set of weights of $r_{\min }$ as before. Borel subgroups of $\mathrm{SL}_{27}$ containing the maximal torus $T_{27}$ (the unique torus stabilizing the weight spaces in $\left.r_{\min }\right)$ are in bijection with orderings of the set $\Lambda_{\min }$; let $B_{27}$ be the Borel defined by the ordering $\lambda>\lambda^{\prime} \Longleftrightarrow h_{\lambda}>h_{\lambda^{\prime}}$. The ordinary deformation ring (again following the notation of [Pat16, Section 4.1]) associated to the Borel $B_{27}$ and the lift $\chi_{T_{27}}: I_{F_{\tilde{v}}} \rightarrow T_{27}\left(\mathbf{Z}_{p}\right)$ of $\left.r_{\min } \circ \bar{\rho}\right|_{G_{F_{\tilde{v}}}}$ given by

$$
\chi_{T_{27}}=r_{\min }\left(\prod_{\alpha \in \Delta} \alpha^{\vee}\left(\epsilon^{\tilde{n}_{v, \alpha}} \chi^{-n_{v, \alpha}}\right)\right)
$$

is formally smooth and receives by the universal property a canonical surjection from $R_{\left.r_{\min } \circ \bar{\rho}\right|_{G_{\tilde{v}}}, H, K-\text { ss }}^{\square}$ for $K=F_{\tilde{v}}(\chi)$ (recall that $\chi$ is the Teichmüller lift of $\left.\bar{\epsilon} \cdot(\bar{\epsilon}[l])^{-1}\right)$, and after inverting $p$ it induces an isomorphism from a unique irreducible component of the source (the equality of dimensions follows from [Kis08] and [Pat16, Section 4.1]). We take $\mathcal{C}_{v}$ to be this component.

- For all other $v \in S$, we take the irreducible component of $R_{\left.r_{\min } \circ \bar{\rho}\right|_{G_{F}}}^{\square}$ parametrizing minimal deformations in the sense of [Pat16, Section 4.4]; using the standard argument, this is a power series ring and induces a unique irreducible component $\mathcal{C}_{v}$ of $R_{\left.r_{\min } \circ \bar{\rho}\right|_{G_{F}}}^{\square}[1 / p]$. 
In each case, we write $R_{\left.r_{\min } \circ \bar{\rho}\right|_{G_{F}}}^{\mathcal{C}_{v}}$ for the associated local lifting ring. Recall [BLGGT14, Section 1.3] that for $v$ not above $p, R_{\left.r_{\min } \circ \bar{\rho}\right|_{G_{\tilde{v}}}}^{\mathcal{C}_{v}}$ is the maximal quotient of $R_{\left.r_{\min } \circ \bar{\rho}\right|_{F_{F_{\tilde{v}}}} ^{\square}}$ that is reduced, $p$-torsion-free, and after inverting $p$ is supported on the component $\mathcal{C}_{v}$. For $v \mid p, R_{\left.r_{\min } \circ \bar{\rho}\right|_{G_{\tilde{v}}}}^{\mathcal{C}_{F_{v}}}$ is in general constructed similarly, but for us it is simply the formally smooth ordinary deformation ring produced by [Pat16, Section 4.1].

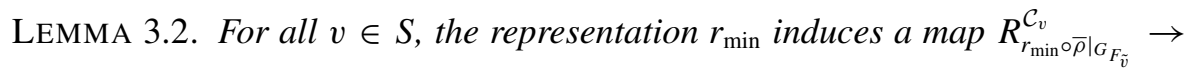
$R_{\left.\bar{\rho}\right|_{G_{\tilde{v}}}}^{\mathcal{P}_{v}}$.

Proof. For $v \mid p$, this follows directly from the definitions once we check that $r_{\min }(B) \subset B_{27}$. Let $\lambda$ be the cocharacter $\sum_{\alpha \in \Delta} \tilde{n}_{v, \alpha} \alpha^{\vee}$. Since all $\tilde{n}_{v, \alpha}$ are positive, $B$ is the locus of $g \in G$ where $\lim _{t \rightarrow 0} \operatorname{Ad}(\lambda(t)) g$ exists. By construction, $B_{27}$ is the locus where $\lim _{t \rightarrow 0} \operatorname{Ad}\left(r_{\min } \circ \lambda(t)\right) g$ exists. The claim follows.

For $v \mid q$, we write $R_{\left.\overline{\rho_{\mid}}\right|_{F_{\tilde{v}}}}^{\mathcal{P}_{v}}$ for the Steinberg lifting ring; recall that under our hypotheses it is a power series ring over $\mathbf{Z}_{p}$ (in $\operatorname{dim}(\mathfrak{g})$ variables). We have surjections

$$
R_{\left.r_{\min } \circ \bar{\rho}\right|_{G_{F}}}^{\square} \rightarrow R_{\left.\bar{\rho}\right|_{G_{F_{\tilde{v}}}}}^{\square} \rightarrow R_{\left.\bar{\rho}\right|_{G_{F_{\tilde{v}}}}}^{\mathcal{P}_{v}} .
$$

Recall that $R_{\left.r_{\min } \circ \bar{\rho}\right|_{G_{\tilde{r}}}}^{\mathcal{C}_{v}}$ is the maximal reduced $p$-torsion-free quotient of $R_{\left.r_{\min } \circ \bar{\rho}\right|_{G_{F}}}^{\square}$ whose $\overline{\mathbf{Q}}_{p}$-points lie on $\mathcal{C}_{v}$. Since $R_{\left.\bar{\rho}\right|_{G_{\tilde{v}}}}^{\mathcal{P}_{v}}$ is a reduced, $p$-torsion-free quotient of $R_{r_{\min } \circ \bar{\rho}_{G_{F}}}^{\square}$, it suffices to show that every $\overline{\mathbf{Q}}_{p}$-point of $R_{\left.\bar{\rho}\right|_{G_{F}}}^{\mathcal{P}_{v}}$ lies on the same irreducible component of $R_{\left.r_{\min } \circ \bar{\rho}\right|_{G_{\tilde{v}}}}^{\square}[1 / p]$ as the representation $\rho_{\tilde{v}}$ (defined above) that characterizes the component $\mathcal{C}_{v}$. As $R_{\left.\bar{\rho}\right|_{G_{F}}}^{\mathcal{P}_{v}}[1 / p]$ is itself irreducible, it suffices to check this for a single $\overline{\mathbf{Q}}_{p}$-point, which we do for the unramified lift $\rho_{\tilde{v}}^{\text {un }}\left(\operatorname{Fr}_{\tilde{v}}\right)=$ $\rho_{G}^{\vee}(q)$. This claim follows from applying [BLGGT14, Lemma 1.3.5] to $\rho_{\tilde{v}}$, whose associated graded for the obvious filtration is canonically identified with $r_{\min } \circ \rho_{\tilde{v}}^{\text {un }}$.

For the other ramified primes $v \in S$, the lemma is evident.

Now consider the global deformation problem (in the sense of [BLGGT14, Section 1.5]; see [CHT08] for details)

$$
\mathcal{S}=\left(F / F^{+}, S,\{\tilde{v}\}_{v \in S}, \mathbf{Z}_{p}, r_{\min } \circ \bar{\rho}, \mu,\left\{\mathcal{C}_{v}\right\}_{v \in S}\right) .
$$

Since $\left.r_{\min } \circ \bar{\rho}\right|_{G_{F}}$ is absolutely irreducible, this deformation functor is prorepresented by some $R_{r_{\text {min }} \circ \bar{\rho}}^{\mathcal{S}}$. 
LEMMA 3.3. The representation $r_{\min }$ induces a surjection $R_{r_{\min } \circ \bar{\rho}}^{\mathcal{S}} \rightarrow R_{\bar{\rho}}^{\mathcal{P}}$.

Proof. There is an induced map $R_{r_{\min } \circ \bar{\rho}}^{\mathcal{S}} \rightarrow R_{\bar{\rho}}^{\mathcal{P}}$ by Lemma 3.2. It is a surjection because the $G_{F^{+}}$-module $\bar{\rho}(\mathfrak{g})$ is a direct summand of $\operatorname{ad}\left(r_{\min } \circ \bar{\rho}\right)$ (indeed, these representations factor through representations of a finite prime-to- $p$ group), so the associated map on tangent spaces is injective; dually, the map on co-tangent spaces is surjective, and we conclude by Nakayama's lemma.

Finally, we can invoke the main results of [BLGGT14] to deduce that $R_{\bar{\rho}}^{\mathcal{P}}$ has a $\overline{\mathbf{Q}}_{p}$-point $\rho$ such that $r_{\min } \circ \rho$ is potentially automorphic:

THEOREM 3.4. For sufficiently general choice of lifts $\tilde{n}_{v, \alpha}$ as in equation (3.1), the representation $\bar{\rho}: G_{F^{+}} \rightarrow{ }^{L} G\left(\mathbf{F}_{p}\right)$ constructed in Proposition 2.8 admits a geometric lift $\rho: G_{F^{+}} \rightarrow{ }^{L} G\left(\overline{\mathbf{Z}}_{p}\right)$ such that:

(1) The Zariski closure of the image of $\rho$ is ${ }^{L} G$.

(2) The composite $\left.r_{\min } \circ \rho\right|_{G_{F}}$ is potentially automorphic in the sense of [BLGGT14].

(3) The composite $\left.r_{\min } \circ \rho\right|_{G_{F}}$ belongs to a compatible system of l-adic representations: there exist a number field $M$ and a strictly pure-in the sense of [BLGGT14, Section 5.1]—compatible system

$$
r_{\lambda}: G_{F} \rightarrow \mathrm{GL}_{27}\left(\bar{M}_{\lambda}\right)
$$

indexed over all finite places $\lambda$ of $M$. In particular, the restriction of $r_{\lambda}$ to $I_{v}$ for $v$ above the auxiliary primes $q \in S_{\mathrm{reg}}$ is unipotent with Jordan blocks of size 1, 9, and 17 as long as $v$ has residue characteristic different from $\lambda$.

Proof. By the proof of [BLGGT14, Theorem 4.3.1] (see especially the last paragraph), $R_{r_{\min } \circ \bar{\rho}}^{\mathcal{S}}$ is a finite $\mathbf{Z}_{p}$-module. Lemma 3.3 then implies that $R_{\bar{\rho}}^{\mathcal{P}}$ is a finite $\mathbf{Z}_{p}$-module. We have already seen in Lemma 3.1 that it has dimension at least one, so we conclude that $R_{\bar{\rho}}^{\mathcal{P}}\left(\overline{\mathbf{Z}}_{p}\right)$ is nonempty. Let $\rho$ be an element of $R_{\bar{\rho}}^{\mathcal{P}}\left(\overline{\mathbf{Z}}_{p}\right)$. Then:

- By [BLGGT14, Theorem 4.5.1], the composite $\left.r_{\min } \circ \rho\right|_{G_{F}}$ is potentially automorphic (in the sense of [BLGGT14]).

- The Zariski closure $G_{\rho}$ of the image of $\left.\rho\right|_{G_{F}}$ is $G$ : by [Pat16, Lemma 7.8], it suffices to show 
- $G_{\rho}$ is reductive;

- $G_{\rho}$ contains a regular unipotent element of $G$; and

- for some $v|p, \rho|_{G_{F_{\tilde{v}}}}$ is $B$-ordinary, and, for all simple roots $\alpha,\left.\alpha \circ \rho\right|_{I_{K}}=\epsilon^{r_{\alpha}}$ for some finite extension $K / F_{\tilde{v}}$ and for distinct integers $r_{\alpha}$.

Reductivity is immediate since $\rho$ is irreducible. The third condition follows by taking the integers $\left\{\tilde{n}_{v, \alpha}\right\}_{\alpha \in \Delta}$ in the definition of the local condition $\mathcal{P}_{v}$ (see the discussion preceding Lemma 3.1) to be sufficiently general. Finally, to show that the image of $\rho$ contains a regular unipotent element, we check that for $v \mid q$, the tame inertia in $\left.\rho\right|_{G_{F_{\tilde{v}}}}$ acts by a regular unipotent. Lemma 3.5 below shows that this would follow from the corresponding claim that $r_{\min } \circ \rho\left(I_{F_{\tilde{v}}}\right)$ contains a unipotent element with Jordan blocks of dimensions 17, 9, and 1. Let $\pi$ be the automorphic representation of $\mathrm{GL}_{27}\left(\mathbf{A}_{F^{\prime}}\right)$, for a suitable finite extension $F^{\prime} / F$, witnessing the potential automorphy of $r_{\min } \circ \rho$, and let $v^{\prime}$ be a place of $F^{\prime}$ above $v$. By local-global compatibility at $l \neq p$ (Proved in general by [Car12], but known for odd dimensional representations by previous work of [HT01, TY07, Shi11]), the (Frobenius semisimple) WeilDeligne representation associated to $\left.r_{\min } \circ \rho\right|_{G_{F_{v^{\prime}}^{\prime}}}$ is isomorphic to the image of $\pi_{v^{\prime}}$ under the local Langlands correspondence. It follows (for example, using [BLGGT14, Lemma 1.3.2(1)]) that $\left.r_{\min } \circ \rho\right|_{G_{F_{\tilde{v}}}}$ lies on a unique irreducible component of $R_{\left.r_{\min } \circ \rho\right|_{F_{\tilde{v}}}}^{\square}$. By construction, $\left.r_{\min } \circ \rho\right|_{G_{F_{\tilde{v}}}}$ and $r_{\min } \circ \rho_{\tilde{v}}$ lie on the same irreducible component $\mathcal{C}_{v}$ of $R_{\left.r_{\min } \circ \bar{\rho}\right|_{G_{\tilde{v}}}}$, and since they both lie on a unique component, [BLGGT14, Lemma 1.3.4(2)] implies their inertial restrictions are isomorphic. The result follows.

The claim that $r_{\min } \circ \rho$ can be put in a compatible system, follows from [BLGGT14, Theorem 5.5.1], and the claim concerning the restriction to $I_{v}$ for $v \mid q$ follows as above from local-global compatibility at $l \neq p$.

In the next section, we show that in fact all members of the compatible system $\left\{r_{\lambda}\right\}_{\lambda}$ have algebraic monodromy group equal to $G$.

Finally, here is the Lie-theoretic lemma promised in the proof of Theorem 3.4.

LEMMA 3.5. Let $G$ be an almost-simple algebraic group over an algebraically closed field of characteristic zero, and let $T$ be a maximal torus in G. Fix a system of simple roots $\Delta=\left\{\alpha_{1}, \ldots, \alpha_{r}\right\}$ of $\mathfrak{g}$ with respect to $T$. Let $\lambda \in X^{\bullet}(T)$ be a nonzero dominant (with respect to $\Delta$ ) weight, with corresponding highest weight representation $r_{\lambda}: G \rightarrow \operatorname{Aut}\left(V_{\lambda}\right)$. Let $N \in \mathfrak{g}$ be a regular nilpotent element. 
Then for any nilpotent element $X \in \mathfrak{g}, r_{\lambda}(X)$ is conjugate to $r_{\lambda}(N)$ if and only if $X$ is regular nilpotent.

Proof. It is well known that the regular nilpotent elements of $\mathfrak{g}$ form a single $G$ orbit, so one direction is clear. By the theorems of Jacobson-Morosov, Kostant, and Mal'cev [CM93, Theorems 3.3.1, 3.4.10, 3.4.12], there is an $\mathfrak{s l}_{2}$-triple $\{X, Y, H\}$ in $\mathfrak{g}$ containing $X$, this triple is unique up to conjugacy, and its conjugacy class is determined by that of $H$. Replacing $\{X, Y, H\}$ by a conjugate, we may assume that $H \in \operatorname{Lie}(T)$ and $\alpha(H) \in\{0,1,2\}$ for all $\alpha \in \Delta$ (see [CM93, Section 3.5]). When we likewise extend $N$ to an $\mathfrak{s l}_{2}$-triple with semisimple element $H_{N}$, and assume after conjugating that $H_{N} \in \operatorname{Lie}(T)$ is dominant, we have $\alpha\left(H_{N}\right)=2$ for all $\alpha \in \Delta$. Since the conjugacy class of $H$ determines that of $X, X$ is regular if and only if $\alpha(H)=2$ for all $\alpha \in \Delta$. We claim that the highest eigenvalue of $H$ on $V_{\lambda}$ is enough to determine whether $X$ is regular. For each weight $\mu$ of $V_{\lambda}$, write

$$
\mu=\sum_{\alpha \in \Delta} b_{\alpha}(\mu) \alpha
$$

for some $b_{\alpha}(\mu) \in \mathbf{Q}$. Because $\lambda$ is the highest weight of $V_{\lambda}$, we have $b_{\alpha}(\lambda) \geqslant$ $b_{\alpha}(\mu)$ for all other weights $\mu$ occurring in $V_{\lambda}$. Assuming that $b_{\alpha}(\lambda)>0$ for all $\alpha$, the maximal eigenvalue of $H$ on $V_{\lambda}$ is thus $\sum_{\alpha \in \Delta} b_{\alpha}(\lambda) \alpha(H)$, and this is strictly less than $\sum_{\alpha \in \Delta} b_{\alpha}(\lambda) \cdot 2$ if $X$ is not regular. If $b_{\alpha}(\lambda) \leqslant 0$, then (by symmetry) $b_{\alpha}(\mu)=0$ for every weight $\mu$ of $V_{\lambda}$. (The sum of all weights occurring in $V_{\lambda}$ is equal to zero because it is invariant under the action of the Weyl group.) But (assuming $\lambda \neq 0$ ) this contradicts the simplicity of $\mathfrak{g}$.

\section{Controlling the image in the compatible system}

Theorem 3.4 provides the existence of a compatible system $\left\{r_{\lambda}\right\}$ of $G_{F}$ representations with the property that the geometric monodromy group at one prime is precisely $E_{6}$. Our goal in this section is to use known properties of compatible systems [LP92] together with the additional properties our compatible system satisfies at the auxiliary primes $S_{\text {reg }}$ to ensure that the monodromy group is $E_{6}$ at all primes $\lambda$.

Let $M$ denote the coefficient field of our compatible system. For a pair of reductive groups $\mathbf{G}_{i}$ over $\bar{M}_{\lambda}$ together with faithful irreducible representations $\rho_{i}$, recall that $\left(\mathbf{G}_{1}, \rho_{1}\right)$ is similar to $\left(\mathbf{G}_{2}, \rho_{2}\right)$ if and only if, for some maximal tori $T_{i} \subset \mathbf{G}_{i}$, the pairs $\left(T_{i},\left.\rho_{i}\right|_{T_{i}}\right)$ are isomorphic (cf. [LP92, Section 5.1]). We would like to compare the following two faithful representations of reductive groups over $\bar{M}_{\lambda}$ : 
(1) The monodromy group $G_{\lambda}$ of $r_{\lambda}$ over $\bar{M}_{\lambda}$ (that is, the Zariski closure of the image of $r_{\lambda}$ over $\bar{M}_{\lambda}$ ) with its tautological representation $r_{\lambda}$,

(2) The group $\left.G\right|_{\bar{M}_{\lambda}}$, where $G$ is (as above) the split simply connected reductive group scheme over $\mathbf{Z}$ of type $E_{6}$, and $r_{\text {min }}$ is the minuscule representation.

LEMMA 4.1. The monodromy group $G_{\lambda}$ for each prime $\lambda$ of $M$ has the following properties:

(1) The component group of $G_{\lambda}$ is trivial.

(2) The rank of $G_{\lambda}$ is 6 .

(3) If $T_{\lambda}$ is a maximal torus in $G_{\lambda}$, and $\left.T\right|_{\bar{M}_{\lambda}}$ is a maximal torus in $\left.G\right|_{\bar{M}_{\lambda}}$, then there is an isomorphism

$$
\left(T_{\lambda},\left.r_{\lambda}\right|_{T_{\lambda}}\right) \simeq\left(\left.T\right|_{\bar{M}_{\lambda}},\left.r_{\min }\right|_{T_{\bar{M}_{\lambda}}}\right) .
$$

(4) If $G_{\lambda}=G_{\lambda}^{\circ}$ acts irreducibly, then $\left(G_{\lambda}, r_{\lambda}\right)$ is similar to $\left(\left.G\right|_{\bar{M}_{\lambda}}, r_{\min }\right)$.

(5) If $G_{\lambda}=G_{\lambda}^{\circ}$ acts irreducibly, then $G_{\lambda}$ is isomorphic to $\left.G\right|_{\bar{M}_{\lambda}}$ and $r_{\lambda}$ is isomorphic to $r_{\min }$.

(6) There exists a unipotent element in the image with Jordan blocks of size 1, 9, and 17.

Proof. The first three properties (1), (2), (3) involve quantities which are constant in a compatible system, cf. [LP92, Propositions 6.12 and 6.14]. We choose $\lambda$ so that $r_{\lambda}=r_{\min } \circ \rho_{F}$ as in Theorem 3.4(1). Then, by Theorem 3.4(1), we deduce that $\left(G_{\lambda}, r_{\lambda}\right) \simeq\left(\left.G\right|_{\bar{M}_{\lambda}}, r_{\min }\right)$ in this case. Claim (4) follows immediately from (3) and the definition of similarity recalled above. Claim (5) follows from Theorem 5.6 of [LP92], noting that $E_{6}$ does not occur in the explicit list of groups which gives rise to any of the basic similarity relations of Section 5 of ibid. The nilpotent operator of the Weil-Deligne representation of $r_{\lambda}$ at the auxiliary prime $v \mid q$ for $q \in S_{\text {reg }}$ decomposes (by Theorem 3.4(3)) into Jordan blocks of size 1, 9, and 17, assuming that the residual characteristic of $q$ is different from that of $\lambda$. Yet $S_{\text {reg }}$ was chosen (for this purpose!) to consist of two primes, so this holds for at least one prime $v$, which gives part (6).

We now show that Conditions (2), (3), and (6) of Lemma 4.1 are sufficient to imply-purely by representation theoretic methods - that $G_{\lambda}$ acts irreducibly, which by Lemma 4.1(5) will prove the claims in Theorem 1.1 concerning the monodromy groups of $\left\{r_{\lambda}\right\}$. In light of the existence of the unipotent element 
whose existence is guaranteed by Lemma 4.1(6), it suffices to show that $G_{\lambda}$ cannot act faithfully on a direct sum of representations of dimension

$$
27=26+1=18+9=17+10=17+9+1
$$

unless $G_{\lambda}=E_{6}$ and the representation is irreducible.

4.1. The Formal Character of $\mathfrak{e}_{6}$. The root lattice $\Phi$ of $E_{6}$ consists of 72 roots; it may be given as $\Phi^{+} \cup \Phi^{-}$, where the positive roots $\Phi^{+}$are given explicitly in $\mathbf{R}^{6}$ by the $2\left(\begin{array}{l}5 \\ 2\end{array}\right)=20$ vectors $e_{i} \pm e_{j}$ for $2 \leqslant i<j \leqslant 6$, and the $2^{4}=\left(\begin{array}{l}5 \\ 0\end{array}\right)+\left(\begin{array}{l}5 \\ 2\end{array}\right)+\left(\begin{array}{l}5 \\ 4\end{array}\right)=16$ vectors

$$
\left(\frac{\sqrt{3}}{2}, \frac{ \pm 1}{2}, \frac{ \pm 1}{2}, \frac{ \pm 1}{2}, \frac{ \pm 1}{2}, \frac{ \pm 1}{2}\right)
$$

where there are an even number of minus signs. If $2 \rho=\sum_{\alpha \in \Phi^{+}} \alpha$, then $\rho=(4 \sqrt{3}$, $4,3,2,1,0)$. The root lattice is not self-dual, but has discriminant 3 . A weight $\mu$ corresponding to a choice of minuscule representation is given by

$$
\mu=\left(\frac{2 \sqrt{3}}{3}, 0,0,0,0,0\right) .
$$

The 27 weights $\Sigma$ of the corresponding minuscule representation may be obtained from $\mu$ from the orbit of the Weyl group; all 27 such weights may be obtained by applying at most 2 reflections in the roots of $\Phi$ to $\mu$. The 27 roots can also be described explicitly in this basis; there are 10 roots of the form $-\sqrt{3} / 3 e_{1} \pm e_{i}$ for $i=2, \ldots, 5$, and $2^{4}=\left(\begin{array}{l}5 \\ 1\end{array}\right)+\left(\begin{array}{l}5 \\ 3\end{array}\right)+\left(\begin{array}{l}5 \\ 5\end{array}\right)=16$ roots of the form

$$
\left(\frac{\sqrt{3}}{6}, \frac{ \pm 1}{2}, \frac{ \pm 1}{2}, \frac{ \pm 1}{2}, \frac{ \pm 1}{2}, \frac{ \pm 1}{2}\right)
$$

where there are an $o d d$ number of minus signs, and finally $\mu$ itself. These three sets (of orders $10+16+1=27$ ) are precisely the orbits under the stabilizer $W_{G, \mu}$ of $\mu$ in the Weyl group $W_{G}$.

LEMMA 4.2. Of the $\left(\begin{array}{c}27 \\ 3\end{array}\right)=2925$ collections of 3 vectors in $\Sigma$, exactly 45 such triples generate a subspace of dimension 2, and they all consist of a triple of weights $\left(\mu, \mu^{\prime}, \mu^{\prime \prime}\right)$ with $\mu+\mu^{\prime}+\mu^{\prime \prime}=0$. 
Proof. This can be checked explicitly using magma given the explicit description of $\Sigma$ given above.

We also recall the following facts (cf. [Lur01]) which are also elementary to verify by direct computation. (These facts are included purely for the reader's enjoyment and are not used in the sequel, except perhaps tangentially in the proof of Lemma 5.2.) If $\Lambda$ is the weight lattice, then $\Sigma$ injects into $V=\Lambda / 2 \Lambda$, which acquires the structure of a quadratic space via the map $q(\mu)=\frac{1}{2}\langle\mu, \mu\rangle$ (note that $\Lambda$ is an even lattice). The pairing $\langle x, y\rangle=q(x+y)-q(x)-q(y)$ is preserved by the Weyl group $W_{G}$, which may be identified with the corresponding orthogonal group $O(V, q)$ [Lur01, Remark 4.3.4]. The triples $\left\{\mu, \mu^{\prime}, \mu^{\prime \prime}\right\}$ of Lemma 4.2 lie inside a maximal isotropic subspace $U \subset V$ of dimension 2. The stabilizer of $U$ (and of a triple) is a subgroup of $W_{G}$ of index 45 , which correspondingly acts transitively on the set of 45 triples. The stabilizer is also isomorphic to the Weyl group of $F_{4}$.

LEMMA 4.3. The quotient of $T_{\lambda}$ which acts faithfully on any subrepresentation of dimension $\geqslant 4$ has rank at least 3 , while $T_{\lambda}$ itself acts faithfully on any subrepresentation of dimension 26. In particular, if $G_{\lambda}$ has a subrepresentation of dimension $\geqslant 4$ or 26 , then the quotient of $G_{\lambda}$ which acts faithfully on this subrepresentation has rank at least 3 or 6 respectively.

Proof. Recall (in the notation of Lemma 4.1) that we have an isomorphism $\left(T_{\lambda},\left.r_{\lambda}\right|_{T_{\lambda}}\right) \simeq\left(\left.T\right|_{\bar{M}_{\lambda}},\left.r_{\min }\right|_{T_{\bar{M}_{\lambda}}}\right)$. Hence it suffices to prove the corresponding claims for the formal character of the minuscule representation of $E_{6}$. For the first claim, it suffices to show that any four weights appearing in the subrepresentation must generate a sublattice of the weight lattice of rank at least 3. Assume otherwise. Then certainly any two of these weights must generate a sublattice of rank at most 2. By Lemma 4.2, such triples of weights are all of the form $\left(\mu, \mu^{\prime}, \mu^{\prime \prime}\right)$ where $\mu+\mu^{\prime}+\mu^{\prime \prime}=0$. But (among four distinct weights) such a relation can hold for at most one such triple. Hence we obtain a contradiction. The second claim follows from the fact that the sum of all 27 weights in $\Sigma$ is zero, while none of the weights in $\Sigma$ is zero.

We now note the following:

LEMMA 4.4. Suppose that $\mathfrak{g}$ is a reductive Lie algebra with a faithful irreducible representation of dimension $d$ for $d \in\{17,26,18\}$. Then $\mathfrak{g}=\mathfrak{h}$ or $\mathfrak{h} \oplus \mathfrak{t}$, where $\mathfrak{h}$ is semisimple and $\mathfrak{t}$ is a rank one torus. Furthermore, assuming that $\mathfrak{h}$ is simple when $d=18$, then $\mathfrak{h}$ is one of the following: 


\begin{tabular}{|c|c|c|}
\hline $\mathfrak{h}$ & $d$ & rank(h) \\
\hline $\mathfrak{s l}_{2}$ & 17 & 1 \\
$\mathfrak{s o}_{17}$ & 17 & 8 \\
$\mathfrak{s l}_{17}$ & 17 & 16 \\
\hline $\mathfrak{s l}_{2}$ & 26 & 1 \\
$\mathfrak{f}_{4}$ & 26 & 4 \\
$\mathfrak{s o}_{13} \times \mathfrak{s l}_{2}$ & 26 & 7 \\
$\mathfrak{s l}_{13} \times \mathfrak{s l}_{2}$ & 26 & 13 \\
$\mathfrak{s p}_{26}$ & 26 & 13 \\
$\mathfrak{s o}_{26}$ & 26 & 13 \\
$\mathfrak{s l}_{26}$ & 26 & 25 \\
\hline $\mathfrak{s l}_{2}$ & 18 & 1 \\
$\mathfrak{s p}_{18}$ & 18 & 9 \\
$\mathfrak{s o}_{18}$ & 18 & 9 \\
$\mathfrak{s l}_{18}$ & 18 & 17 \\
\hline \multicolumn{2}{|r}{}
\end{tabular}

Proof. It suffices to classify all small (of dimension at most 27) representations of the simple Lie groups; one may (and we did) compute these using the Weyl character formula. One can also deduce this result immediately for groups of rank $\leqslant 8$ by consulting [MP81, Table 1, pages 25-83]. Groups of rank $>8$ can also be handled directly by hand, but they also play no role in the argument below because we are always able to immediately dismiss any groups of rank $>6=$ $\operatorname{rank}\left(E_{6}\right)$.

Let us now return to the possible cases in which our 27-dimensional Galois representation is reducible, and consider the corresponding monodromy groups. Suppose there is a constituent of dimension 17. The rank must be bounded by 6. From Lemma 4.4, it follows that the Lie algebra of the monodromy group on this summand must be $\mathfrak{s l}_{2}$ or $\mathfrak{s l}_{2} \times \mathfrak{t}$. But the rank of these algebras is at most 2, which violates Lemma 4.3. Suppose there is a constituent of dimension 26. Then by Lemma 4.3, the rank of the Lie algebra of this representation is exactly 6 , and hence the rank of $\mathfrak{h}$ with $\mathfrak{g}=\mathfrak{h}$ or $\mathfrak{h} \times \mathfrak{t}$ is 6 or 5 . Since there are no such groups of this rank with irreducible representations of dimension 26 by Lemma 4.4, we once more derive a contradiction. Hence the only remaining possibility is that the 27- dimensional representation decomposes into two irreducible pieces of dimensions 9 and 18, corresponding to a decomposition of weights $\Sigma=\Sigma_{9} \cup \Sigma_{18}$. On the other hand, we know that the 18-dimensional representation must have a unipotent element with Jordan blocks of size $1+17$. The tensor product of two Jordan blocks of size $m$ and $n$ with $n \leqslant m$ decomposes into blocks of 
size $n+m-1, n+m-3, \ldots, n-m+1$. In particular, it must be the case that the 18-dimensional representation does not factor into a product of smaller dimensional representations, since otherwise there could not be a Jordan block of a unipotent element of size as larger as 17 . Hence the monodromy group on this representation must have a simple Lie algebra (up to a torus). Again, we deduce from Lemma 4.4 and using rank considerations that $\mathfrak{g}=\mathfrak{s l}_{2}$ or $\mathfrak{s l}_{2} \times \mathfrak{t}$, once more contradicting Lemma 4.3.

We conclude:

Corollary 4.5. Let $\left\{r_{\lambda}: G_{F} \rightarrow \mathrm{GL}_{27}\left(\bar{M}_{\lambda}\right)\right\}_{\lambda}$ be the compatible system produced in Theorem 3.4. Then for all $\lambda$, the Zariski closure of the image of $r_{\lambda}$ is isomorphic to $\left.G\right|_{M_{\lambda}}$ under the minuscule representation $r_{\mathrm{min}}$.

\section{5. $E_{6}$ motives over CM fields}

We conclude by showing that $r_{\lambda}$ is a subrepresentation of the cohomology of some smooth projective variety over $F$, thus completing the proof of Theorem 1.1. Note that, according to the Tate conjecture, we expect that such a compatible family should be cut out by correspondences over $F$, and thus arise from a motive $M$ over $F$. We do not have any idea how to prove this. On the other hand, we do know that the compatible family $r_{\lambda}$ becomes automorphic over a CM extension $H / F / \mathbf{Q}$, and (since we are in highly regular weight), using standard methods combined with the work of Shin [Shil1], one can associate a motive $M$ over $H$ whose associated $p$-adic Galois representations $\left\{\left.r_{\lambda}\right|_{G_{H}}\right\}$ have monodromy group $E_{6}$. More precisely, we should say that one expects to be able to associate such a motive where the correspondences cutting out $M$ arise from Hecke operators. In practice, we take a shortcut and deduce from [Shi11] the weaker claim that the Galois representations over $G_{H}$ (and thus over $G_{F}$ by restriction of scalars) came from cohomology. We apologize for the omission and leave it as an exercise to the more responsible reader to write down the correct argument.

To set up all the required notation would be quite cumbersome, so we simply use the notation of [Shi11], giving precise references to where the relevant terms are defined. We hope that a reader with a copy of [Shi11] at hand can easily follow this argument. Fix an isomorphism $\iota_{l}: \overline{\mathbf{Q}}_{l} \stackrel{\sim}{\rightarrow} \mathbf{C}$; it is implicit in all of the constructions of [Shi11]. By Theorem 3.4, there is a CM extension $H / F$ and a cuspidal automorphic representation $\Pi^{0}$ of $\mathrm{GL}_{27}\left(\mathbf{A}_{H}\right)$ such that

- $\left(\Pi^{0}\right)^{\vee} \cong\left(\Pi^{0}\right)^{c}$.

- $\left.R_{l}\left(\Pi^{0}\right) \cong r_{\lambda}\right|_{G_{H}} \cdot \epsilon^{(1-n) / 2}$, in the notation of [Shi11, Theorem 7.5]. (Note that $n$ is odd, and we have taken into account a standard twist in the association 
of Galois representations to cohomological automorphic representations, as in [Shi11]; the relation between the conjugate self-dualities on the automorphic and Galois sides is explicitly stated in [BLGGT14, Theorem 2.1.1]. Of course it will suffice to show this Tate twist of $r_{\lambda}$ appears in cohomology.)

- $\left[H^{+}: \mathbf{Q}\right] \geqslant 2$, and $H$ contains a quadratic imaginary field (we can simply enlarge an initial choice of $H$ to ensure these conditions).

Set $n=27$, for ease of reference to [Shi11]; we recall the construction of $R_{l}\left(\Pi^{0}\right)$ and see as a result that after some further base change that there is an explicit description of this Galois representation in the cohomology of a unitary similitude group Shimura variety. We begin with two reductions. Let $E$ be an imaginary quadratic field not contained in $H$ satisfying the four bulleted conditions in Step (II) of the proof of [Shi11, Theorem 7.5]. Replace $H$ by $H E$ and $\Pi^{0}$ by $\mathrm{BC}_{H E / H}\left(\Pi^{0}\right)$. Then having made this replacement the triple $\left(E, H, \Pi^{0}\right)$ satisfies the six bulleted conditions at the beginning of Step (I) of the proof of [Shi11, Theorem 7.5]. Let $H^{\prime}$ be an imaginary quadratic extension of $H^{+}$satisfying the three bulleted conditions (defining the set denoted $\mathcal{F}(H)$-but note our $H$ is Shin's $F$ ) in Step (I) of [Shi11, Theorem 7.5]. Then replace $H$ by $H H^{\prime}$ and $\Pi^{0}$ by $\mathrm{BC}_{H H^{\prime} / H}\left(\Pi^{0}\right)$. Again having made this replacement, [Shi11, Proposition 7.4] now applies to the triple $\left(E, H, \Pi^{0}\right)$. There is a Hecke character $\psi$ of $\mathbf{A}_{E}^{\times} / E^{\times}$ such that, setting $\Pi=\psi \otimes \Pi^{0}$ (an automorphic representation of the group $\mathbb{G}_{n}(\mathbf{A}) \cong \mathrm{GL}_{1}\left(\mathbf{A}_{E}\right) \times \mathrm{GL}_{n}\left(\mathbf{A}_{H}\right)$ of [Shi11, Section 3.1]), we have (in the notation of [Shi11, Corollary 6.8])

$$
R_{l}\left(\Pi^{0}\right):=R_{l}^{\prime}(\Pi):=\left.\widetilde{R}_{l}^{\prime}(\Pi) \otimes \operatorname{rec}_{l, l_{l}}\left(\psi^{c}\right)\right|_{G_{H}},
$$

where (see [Shi11, 5.5, 6.23], and note that the group $G$ no longer denotes $E_{6}$, but rather the unitary similitude group defined in [Shi11, Section 5.1]!)

$$
C_{G} \cdot \widetilde{R}_{l}^{\prime}(\Pi)=\sum_{\pi^{\infty} \in \mathcal{R}_{l}(\Pi)} R_{\xi, l}^{n-1}\left(\pi^{\infty}\right)^{\mathrm{ss}} .
$$

In our case, the integer $C_{G}=\tau(G) \operatorname{ker}^{1}(\mathbf{Q}, G)$ (defined in [Shi11, Theorem 6.1]) is 2: this is explained in [Tay12, pages $411-412$ ]. Moreover, $\widetilde{R}_{l}^{\prime}(\Pi)$ is irreducible (since the image of $\left.r_{\lambda}\right|_{\Gamma_{H}}$ is Zariski-dense in $E_{6}$ ), and $\mathcal{R}_{l}(\Pi)$ in fact contains at least two elements: following [Tay12, page 413], there will be two automorphic representations of $G\left(\mathbf{A}_{\mathbf{Q}}\right)$, differing by a twist but having isomorphic base changes to $\mathrm{GL}_{n}\left(\mathbf{A}_{H}\right)$, that contribute to $\mathcal{R}_{l}(\Pi)$ (these are denoted $\tilde{\pi}$ and $\tilde{\pi} \otimes$ $\left(\delta_{A / \mathbf{Q}} \circ v\right)$ in [Tay12, page 413]). We fix one such $\tilde{\pi}^{\infty} \in \mathcal{R}_{l}(\Pi)$. It follows that $\widetilde{R}_{l}^{\prime}(\Pi) \cong R_{\xi, l}^{n-1}\left(\widetilde{\pi}^{\infty}\right)$ (no semisimplification necessary because these are irreducible representations). 
Now recall the decomposition [Shi11, 5.5]

$$
H^{n-1}\left(\mathrm{Sh}, \mathcal{L}_{\xi}\right)=\bigoplus_{\pi^{\infty}} \pi^{\infty} \otimes R_{\xi, l}^{n-1}\left(\pi^{\infty}\right) .
$$

At some finite level $U$, we deduce that $\widetilde{R}_{l}^{\prime}(\Pi)$ is contained in $H^{n-1}\left(\operatorname{Sh}_{U} \times H \bar{H}, \mathcal{L}_{\xi}\right)$ (it is even a direct summand cut out by Hecke operators). Finally, letting $\mathcal{A}_{U} \rightarrow$ $\mathrm{Sh}_{U}$ denote the universal abelian scheme (arising from the PEL moduli problem), and letting $\mathcal{A}_{U}^{(m)}$ denote its $m$-fold fibre product over $\mathrm{Sh}_{U}$ (for any integer $m \geqslant 1$ ), then there are integers $m_{\xi}$ and $t_{\xi}$ (see [HT01, page 98]) such that

$$
H^{n-1}\left(\mathrm{Sh}_{U} \times_{H} \bar{H} ; \mathcal{L}_{\xi}\right) \cong \varepsilon \cdot H^{n-1+m_{\xi}}\left(\mathcal{A}_{U}^{\left(m_{\xi}\right)} \times_{H} \bar{H}, \overline{\mathbf{Q}}_{l}\right)\left(t_{\xi}\right),
$$

where $\varepsilon$ is a suitable idempotent projector. We recall that our assumption $\left[\mathrm{H}^{+}\right.$: Q] $\geqslant 2$ implies that $\mathrm{Sh}_{U}$, and therefore $\mathcal{A}_{U}^{\left(m_{\xi}\right)}$, are smooth projective varieties over $H$. Thus $\left.\left.\left(r_{\lambda} \cdot \epsilon^{(1-n) / 2}\right)\right|_{G_{H}} \otimes \operatorname{rec}_{l, l}\left(\psi^{c}\right)^{-1}\right|_{G_{H}}$ is a subrepresentation of the cohomology of the smooth projective variety $\mathcal{A}_{U}^{\left(m_{\xi}\right)}$ over $H$. Possibly replacing $H$ by a finite extension, we can find a product of $\mathrm{CM}$ abelian varieties $A / H$ such that $\left.\operatorname{rec}_{l, l_{l}}\left(\psi^{c}\right)\right|_{G_{H}}$ is a subrepresentation of $H^{i}\left(A \times_{H} \bar{H}, \overline{\mathbf{Q}}_{l}\right)(j)$ for some integers $i$ and $j$ [DMOS82, IV. Proposition D.1]. We conclude that $\left.r_{\lambda}\right|_{G_{H}}$ is a subrepresentation of $H^{r}\left(X \times_{H} \bar{H}, \overline{\mathbf{Q}}_{l}\right)(s)$ for some smooth projective variety $X / H$ (namely, $X=A \times_{H} \mathcal{A}_{U}^{\left(m_{\xi}\right)}$ ). By Frobenius reciprocity and irreducibility of $r_{\lambda}, r_{\lambda}$ (as $G_{F}$-representation) is a subrepresentation of $\operatorname{Ind}_{G_{H}}^{G_{F}}\left(H^{r}\left(X \times_{H} \bar{H}, \overline{\mathbf{Q}}_{l}\right)(s)\right)$. This induction is just the cohomology of $X$ regarded as a variety over $F$ (that is, via $X \rightarrow \operatorname{Spec}(H) \rightarrow \operatorname{Spec}(F))$, so the proof of Theorem 1.1 is complete.

REMARK 5.1. One may reasonably ask whether there exist $E_{6}$ motives (or strongly compatible systems) over $\mathbf{Q}$. We do not know the answer. There is, however, a technical obstruction for applying the methods of this paper. To use automorphic methods, the Hodge-Tate weights of $r_{\min } \circ \rho_{\lambda}$ must be distinct. However, there cannot exist such a compatible system over $\mathbf{Q}$ (or any totally real field), since, by Corollary 5.4.3 of [BLGGT14], this would imply that the trace of complex conjugation representation must be \pm 1 , and, as the following lemma shows, there are no such involutions in $E_{6}(\mathbf{C}) \subset \mathrm{GL}_{27}(\mathbf{C})$.

LEMMA 5.2. There are two conjugacy classes of nontrivial involutions in $E_{6}(\mathbf{C})$ with traces -5 and 3 respectively.

Proof. This lemma is 'well known' in the sense that at least one of the authors of this paper claims to know it. In lieu of a reference, we give the following computational proof, which we explain in as conceptual terms as possible. 
Let $X_{\bullet}=X_{\bullet}(T)$ denote the cocharacter group of $G$, and $X^{\bullet}=X^{\bullet}(T)$ the character group. We may assume any involution lies in $T$ and thus can be considered as an element of $X_{\bullet} \otimes \mu_{2} \simeq\left(X^{\bullet} / 2 X^{\bullet}\right)^{\vee}$. We may identify the latter group with the $\mathbf{F}_{2}$-dual $V^{\vee}$ of $V=\Lambda / 2 \Lambda$. This is a quadratic space, thus self-dual, and so there is a $W_{G}$-equivariant isomorphism $V^{\vee} \simeq V$. There are $63=2^{6}-1$ nonzero elements of $V$. These are divide into two sets depending on whether the vectors are isotropic or not. The action of $W_{G}$ correspondingly has two orbits of size 27 and 36 respectively. This is almost true by pure thought, since the orbits must have order greater than 2 ( $V$ is an irreducible $W_{G}$-representation) and the only nontrivial permutation representations of $W_{G}$ of degree less than 40 and greater than 2 are of orders 27 and 36 respectively [ $\mathbf{C C N}^{+} \mathbf{8 5}$ ]. In fact, one can identify these orbits of size 27 and 36 are precisely the images of the set of weights and positive roots, respectively. If $\mu$ is the highest weight vector corresponding to $r_{\min }$, let $W_{G, \mu}$ the stabilizer of $\mu$ in $W_{G}$. Since $r_{\min }$ is minuscule, the corresponding weights are precisely those of the form $w \mu$ for $w \in W_{G} / W_{G, \mu}$. Hence the trace of an involution corresponding to $v^{\vee}$ turns out to be equal to

$$
\sum_{w \in W_{G} / W_{G, \mu}}(-1)^{\left\langle v^{\vee}, w \mu\right\rangle} .
$$

By computing this sum explicitly over all $v^{\vee}$, we see that the traces of the two nontrivial involutions in the minuscule representation are -5 and 3 for precisely 27 and 36 of the $v^{\vee}$ respectively. For example, for the first sum, we have already noted that the stabilizer $W_{\mu}$ acts on the roots with orbits of size 1,10 , and 16 respectively given explicitly before Lemma 4.2; the corresponding signs in the sum are $+1,+1$, and -1 respectively, and hence the sum is $1+10-16=-5$. The second sum can be computed directly from the first by averaging over the $W_{G}$ action and then using the fact that, for any fixed $v^{\vee}$, one has

$$
\sum_{v \in V}(-1)^{\left\langle v^{\vee}, v\right\rangle}=0 .
$$

Alternatively, if $W_{G, \alpha}$ is the stabilizer of the image of a root $\alpha \in V$ (and hence of index 36 in $W_{G}$ ), then the orbits of $W_{G, \alpha}$ on the weights are of size 15 and 12 , and the corresponding signs are +1 and -1 and the trace is $15-12=+3$.

As a confirmation of these numbers, we can appeal to the following variation. There exists a cover (the extended Weyl group of Tits [Tit66]) $\widetilde{W}_{G}$ of $W_{G}$ by $V=(\mathbf{Z} / 2 \mathbf{Z})^{6}$ which lives as a subgroup of $E_{6}(\mathbf{C})$. The group has an explicit description as the $\mathbf{Z}$-points of the normalizer $N$ of a (split) maximal torus $T$ of $G$. The group $\widetilde{W}_{G}$ admits a 27 - dimensional representation via the minuscule representation of $E_{6}$ which turns out to be absolutely irreducible. Then one can check (using magma) from the character table that the two conjugacy classes of 
involution in $V=\operatorname{ker}\left(\widetilde{W}_{G} \rightarrow W_{G}\right)$ (of orders 27 and 36 respectively) have traces -5 and 3 under both irreducible 27-dimensional representations of $\widetilde{W}_{G}$.

\section{Complements}

We note the following application of Theorem 1.1 to the inverse Galois problem. Let us write $E_{6}^{\mathrm{sc}}\left(\mathbf{F}_{l}\right)$ for the $\mathbf{F}_{l}$-points of the (simply connected) form of $E_{6}$ that we have been considering. The group $E_{6}^{\mathrm{sc}}\left(\mathbf{F}_{l}\right)$ is the Schur cover of the simple Chevalley group of type $E_{6}$ (with Schur multiplier of order $(3, l-1)$ ), which we denote below by $E_{6}\left(\mathbf{F}_{l}\right)$. The groups $E_{6}^{\mathrm{sc}}\left(\mathbf{F}_{l}\right)$ and $E_{6}^{\mathrm{sc}}\left(\mathbf{F}_{l}\right) .2$ are known to occur as Galois groups over $\mathbf{Q}$ for $p \equiv 4,5,6,9,16,17 \bmod 19$ (these are primes of order 9 in $\mathbf{F}_{19}^{\times}$) by [Mal88, Theorem 2.3] as a consequence of the rigidity method. In contrast, we can prove that $E_{6}^{\mathrm{sc}}\left(\mathbf{F}_{l}\right) .2$ is a Galois group over $\mathbf{Q}$ for a positive density of primes $\equiv 1 \bmod 19$ (by taking $E=\mathbf{Q}\left(\zeta_{19}\right)$ below).

COROLlary 6.1. Let $F$ be an imaginary quadratic field. Then, for a set $S$ of primes $l$ of positive density, there exists a number field $L / \mathbf{Q}$ containing $F$ with $\operatorname{Gal}(L / \mathbf{Q})={ }^{L} G\left(\mathbf{F}_{l}\right)=E_{6}^{\mathrm{sc}}\left(\mathbf{F}_{l}\right) .2$ and $\operatorname{Gal}(L / F)=E_{6}^{\mathrm{sc}}\left(\mathbf{F}_{l}\right)$. Moreover, one may assume that all the primes in $S$ split completely in any finite extension $E / \mathbf{Q}$.

Proof. We combine the previous result with Theorem 3.17 of [Lar95], to deduce that $E_{6}^{\mathrm{sc}}\left(\mathbf{F}_{l}\right)$ is the Galois group of the kernel of $\rho_{\lambda}$ over $F$ for a relative density one set of primes $l$ which split completely in the coefficient field $M$ of the compatible system. In particular, the intersection of $S$ with the set of primes split completely in $E / \mathbf{Q}$ has positive density. Since the representation $\bar{r}_{\lambda}: G_{F} \rightarrow \mathrm{GL}_{27}\left(\mathbf{F}_{l}\right)$ is, by construction, conjugate self-dual, it extends to a representation of $G_{\mathbf{Q}}$ to $\mathcal{G}_{27}\left(\mathbf{F}_{l}\right)$ whose kernel therefore has Galois group ${ }^{L} G\left(\mathbf{F}_{l}\right)$.

REMARK 6.2. Note that Corollary 6.1 remains true if one replaces $E_{6}^{\text {sc }}\left(\mathbf{F}_{l}\right) .2$ and $E_{6}^{\text {sc }}\left(\mathbf{F}_{l}\right)$ by $E_{6}\left(\mathbf{F}_{l}\right) .2$ and $E_{6}\left(\mathbf{F}_{l}\right)$, for the obvious reason that the latter groups are quotients of the former groups.

\section{Acknowledgements}

This work traces its origins to a discussion following a number theory seminar given by one of us (S.P.) at the University of Chicago in February 2016, where all the authors of this paper were in attendance. We would like to thank Sug Woo Shin for answering questions related to his paper [Shi11] relevant for the discussion in Section 5. Finally, we would like to thank the referee for a number of useful suggestions which helped to improve this manuscript. 
G.B. was supported in part by NSF postdoctoral fellowship DMS-1503047, F.C. was supported in part by NSF Grant DMS-1701703, M.E. was supported in part by NSF Grant DMS-1601871, K.M.P. was supported in part by NSF Grant DMS1803623, S.P. was supported in part by NSF Grant DMS-1700759.

\section{References}

[AH16] J. Adams and X. He, 'Lifting of elements of Weyl groups', 2016, MR 3659328.

[BLGGT14] T. Barnet-Lamb, T. Gee, D. Geraghty and R. Taylor, 'Potential automorphy and change of weight', Ann. of Math. (2) 179(2) (2014), 501-609.

[Car12] A. Caraiani, 'Local-global compatibility and the action of monodromy on nearby cycles', Duke Math. J. 161(12) (2012), 2311-2413.

[CCN $\left.{ }^{+} 85\right]$ J. H. Conway, R. T. Curtis, S. P. Norton, R. A. Parker and R. A. Wilson, Atlas of Finite Groups (Oxford University Press, Eynsham, 1985), Maximal subgroups and ordinary characters for simple groups, With computational assistance from J. G. Thackray.

[CHT08] L. Clozel, M. Harris and R. Taylor, 'Automorphy for some $l$-adic lifts of automorphic mod $l$ Galois representations', Publ. Math. Inst. Hautes Études Sci. (108) (2008), 1-181. With Appendix A, summarizing unpublished work of Russ Mann, and Appendix B by Marie-France Vignéras, MR 2470687.

[CM93] D. H. Collingwood and W. M. McGovern, Nilpotent Orbits in Semisimple Lie Algebras, Van Nostrand Reinhold Mathematics Series (Van Nostrand Reinhold Co., New York, 1993).

[DMOS82] P. Deligne, J. S. Milne, A. Ogus and K.-y. Shih, Hodge Cycles, Motives, and Shimura Varieties, Lecture Notes in Mathematics, 900 (Springer, Berlin, 1982).

[DR10] M. Dettweiler and S. Reiter, 'Rigid local systems and motives of type $G_{2}$ ', Compos. Math. 146(4) (2010), 929-963. With an appendix by Michael Dettweiler and Nicholas M. Katz.

[FM95] J.-M. Fontaine and B. Mazur, 'Geometric Galois representations', in Elliptic Curves, Modular Forms, \& Fermat's Last Theorem (Hong Kong, 1993), Ser. Number Theory, I (Int. Press, Cambridge, MA, 1995), 41-78.

[Gro00] B. H. Gross, 'On minuscule representations and the principal $\mathrm{SL}_{2}$ ', Represent. Theory 4 (2000), 225-244 (electronic).

[HT01] M. Harris and R. Taylor, The Geometry and Cohomology of Some Simple Shimura Varieties, Annals of Mathematics Studies, 151 (Princeton University Press, Princeton, NJ, 2001), With an appendix by Vladimir G. Berkovich.

[Kis08] M. Kisin, 'Potentially semi-stable deformation rings', J. Amer. Math. Soc. 21(2) (2008), 513-546.

[KW09a] C. Khare and J.-P. Wintenberger, 'Serre's modularity conjecture. I', Invent. Math. 178(3) (2009), 485-504.

[KW09b] C. Khare and J.-P. Wintenberger, 'Serre's modularity conjecture. II', Invent. Math. 178(3) (2009), 505-586.

[Lar95] M. Larsen, 'Maximality of Galois actions for compatible systems', Duke Math. J. 80(3) (1995), 601-630.

[LP92] M. Larsen and R. Pink, 'On $l$-independence of algebraic monodromy groups in compatible systems of representations', Invent. Math. 107(3) (1992), 603-636.

[Lur01] J. Lurie, 'On simply laced Lie algebras and their minuscule representations', Comment. Math. Helv. 76(3) (2001), 515-575. 
[Mal88] G. Malle, 'Exceptional groups of Lie type as Galois groups', J. reine angew. Math. 392 (1988), 70-109.

[MP81] W. G. McKay and J. Patera, Tables of Dimensions, Indices, and Branching Rules for Representations of Simple Lie Algebras, Lecture Notes in Pure and Applied Mathematics, 69 (Marcel Dekker, Inc., New York, 1981).

[NSW08] J. Neukirch, A. Schmidt and K. Wingberg, Cohomology of Number Fields, 2nd edn, Grundlehren der Mathematischen Wissenschaften [Fundamental Principles of Mathematical Sciences], 323 (Springer, Berlin, 2008).

[Pat16] S. Patrikis, 'Deformations of Galois representations and exceptional monodromy', Invent. Math. 205(2) (2016), 269-336.

[Pat17] S. Patrikis, 'Deformations of Galois representations and exceptional monodromy, II: raising the level', Math. Ann. 368(3-4) (2017), 1465-1491.

[Ram02] R. Ramakrishna, 'Deforming Galois representations and the conjectures of Serre and Fontaine-Mazur', Ann. of Math. (2) 156(1) (2002), 115-154.

[Rob17] D. Roberts, 'Newforms with rational coefficients', Ramanujan J. (2017), to appear, MR 3826758.

[Sch90] A. J. Scholl, 'Motives for modular forms', Invent. Math. 100(2) (1990), 419-430.

[Ser94] J.-P. Serre, 'Propriétés conjecturales des groupes de Galois motiviques et des représentations $l$-adiques', in Motives (Seattle, WA, 1991), Proc. Sympos. Pure Math., 55 (Amer. Math. Soc., Providence, RI, 1994), 377-400.

[Ser08] J.-P. Serre, Topics in Galois Theory, 2nd edn, Research Notes in Mathematics, 1 (A. K. Peters, Ltd., Wellesley, MA, 2008), With notes by Henri Darmon.

[Shi11] S. W. Shin, 'Galois representations arising from some compact Shimura varieties', Ann. of Math. (2) 173(3) (2011), 1645-1741.

[Tay12] R. Taylor, 'The image of complex conjugation in $l$-adic representations associated to automorphic forms', Algebra Number Theory 6(3) (2012), 405-435.

[TY07] R. Taylor and T. Yoshida, 'Compatibility of local and global Langlands correspondences', J. Amer. Math. Soc. 20(2) (2007), 467-493.

[Tit66] J. Tits, 'Normalisateurs de tores. I. Groupes de Coxeter étendus', J. Algebra 4 (1966), 96-116.

[Yun14] Z. Yun, 'Motives with exceptional Galois groups and the inverse Galois problem', Invent. Math. 196(2) (2014), 267-337. 